\title{
A breakdown of injectivity for weighted ray transforms in multidimensions
}

\author{
F. O. Goncharov and R. G. Novikov
}

\begin{abstract}
We consider weighted ray-transforms $P_{W}$ (weighted Radon transforms along oriented straight lines) in $\mathbb{R}^{d}, d \geq 2$, with strictly positive weights $W$. We construct an example of such a transform with non-trivial kernel in the space of infinitely smooth compactly supported functions on $\mathbb{R}^{d}$. In addition, the constructed weight $W$ is rotation-invariant continuous and is infinitely smooth almost everywhere on $\mathbb{R}^{d} \times \mathbb{S}^{d-1}$. In particular, by this construction we give counterexamples to some well-known injectivity results for weighted ray transforms for the case when the regularity of $W$ is slightly relaxed. We also give examples of continous strictly positive $W$ such that $\operatorname{dim} \operatorname{ker} P_{W} \geq n$ in the space of infinitely smooth compactly supported functions on $\mathbb{R}^{d}$ for arbitrary $n \in \mathbb{N} \cup\{\infty\}$, where $W$ are infinitely smooth for $d=2$ and infinitely smooth almost everywhere for $d \geq 3$.
\end{abstract}

\section{Introduction}

We consider the weighted ray transforms $P_{W}$ defined by

$$
\begin{aligned}
& P_{W} f(x, \theta)=\int_{\mathbb{R}} W(x+t \theta, \theta) f(x+t \theta) d t, \quad(x, \theta) \in T \mathbb{S}^{d-1}, d \geq 2, \\
& T \mathbb{S}^{d-1}=\left\{(x, \theta) \in \mathbb{R}^{d} \times \mathbb{S}^{d-1}: x \theta=0\right\},
\end{aligned}
$$

where $f=f(x), W=W(x, \theta), x \in \mathbb{R}^{d}, \theta \in \mathbb{S}^{d-1}$. Here, $W$ is the weight, $f$ is a test function on $\mathbb{R}^{d}$. In addition, we interpret $T \mathbb{S}^{d-1}$ as the set of all rays in $\mathbb{R}^{d}$. As a ray $\gamma$ we understand a straight line with fixed orientation. If $\gamma=\gamma(x, \theta),(x, \theta) \in T \mathbb{S}^{d-1}$, then

$$
\gamma(x, \theta)=\left\{y \in \mathbb{R}^{d}: y=x+t \theta, t \in \mathbb{R}\right\} \quad \text { (up to orientation) }
$$

Key words and phrases: Radon transforms, ray transforms, integral geometry, injectivity, non-injectivity.

2010 Mathematics Subject Classification: 44A12, 53C65, 65R32. 
where $\theta$ gives the orientation of $\gamma$.

We assume that

$$
W=\bar{W} \geq c>0, \quad W \in L^{\infty}\left(\mathbb{R}^{d} \times \mathbb{S}^{d-1}\right),
$$

where $\bar{W}$ denotes the complex conjugate of $W, c$ is a constant.

Note also that

$$
P_{W} f(x, \theta)=\int_{\gamma} W(y, \gamma) f(y) d y, \quad \gamma=\gamma(x, \theta),
$$

where

$$
W(y, \gamma)=W(y, \theta) \quad \text { for } y \in \gamma, \gamma=\gamma(x, \theta),(x, \theta) \in T \mathbb{S}^{d-1} .
$$

The aforementioned transforms $P_{W}$ arise in various domains of pure and applied mathematics; see [LB73], [TM80], [Q83], [Be84], [MQ85], [Fi86], [BQ87], [Sh92], [Kun92], [BQ93], [B93], [Sh93], [KLM95], [Pa96], [ABK98], [Na01], [N02a], [N02b], [BS04], [Bal09], [Gi10], [BJ11], [PG13], [N14], [I16], [Ng17] and references therein.

In particular, the related results are the most developed for the case when $W \equiv 1$. In this case $P_{W}$ is reduced to the classical ray-transform $P$ (Radon transform along straight lines). The transform $P$ arises, in particular, in the $\mathrm{X}$-ray transmission tomography. We refer to [R17], [J38], [C64], [GGG82], [H01], [Na01] and references therein in connection with basic results for this classical case.

At present, many important results on transforms $P_{W}$ with other weights $W$ satisfying (1.4) are also known; see the publications mentioned above with nonconstant $W$ and references therein.

In particular, assuming (1.4) we have the following injectivity results.

Injectivity 1. (see [Fi86]) Suppose that $d \geq 3$ and $W \in C^{2}\left(\mathbb{R}^{d} \times \mathbb{S}^{d-1}\right)$. Then $P_{W}$ is injective on $L_{0}^{p}\left(\mathbb{R}^{d}\right)$ for $p>2$, where $L_{0}^{p}$ denotes compactly supported functions from $L^{p}$.

Injectivity 2. (see [MQ85]) Suppose that $d=2, W \in C^{2}\left(\mathbb{R}^{2} \times \mathbb{S}^{1}\right)$ and

$$
0<c_{0} \leq W, \quad\|W\|_{C^{2}\left(\mathbb{R}^{2} \times \mathbb{S}^{1}\right)} \leq N,
$$

for some constants $c_{0}$ and $N$. Then, for any $p>2$, there is $\delta=\delta\left(c_{0}, N, p\right)>0$ such that $P_{W}$ is injective on $L^{p}\left(B\left(x_{0}, \delta\right)\right)$ for any $x_{0} \in \mathbb{R}^{2}$, where

$$
\begin{aligned}
& L^{p}\left(B\left(x_{0}, \delta\right)\right)=\left\{f \in L^{p}\left(\mathbb{R}^{2}\right): \operatorname{supp} f \subset \bar{B}\left(x_{0}, \delta\right)\right\}, \\
& \bar{B}\left(x_{0}, \delta\right)=\left\{x \in \mathbb{R}^{2}:\left|x-x_{0}\right| \leq \delta\right\}
\end{aligned}
$$


Injectivity 3. (see [Q83]) Suppose that $d=2, W \in C^{1}\left(\mathbb{R}^{2} \times \mathbb{S}^{1}\right)$ and $W$ is rotation invariant (see formula (2.18) below). Then $P_{W}$ is injective on $L_{0}^{p}\left(\mathbb{R}^{2}\right)$ for $p \geq 2$.

In a similar way with [Q83], we say that $W$ is rotation invariant if and only if

$$
\begin{aligned}
& W(x, \gamma) \text { is independent of the orientation of } \gamma \\
& W(x, \gamma)=W(A x, A \gamma) \text { for } x \in \gamma, \gamma \in T \mathbb{S}^{d-1}, A \in O(d),
\end{aligned}
$$

where $T \mathbb{S}^{d-1}$ is defined in (1.2), $O(d)$ denotes the group of orthogonal transformations of $\mathbb{R}^{d}$.

Note also that property (1.9) can be rewritten in the form (2.18), (2.19) or (2.20), (2.21); see Section 2.

Injectivity 4. (see [BQ87]) Suppose that $d=2, W$ is real-analytic on $\mathbb{R}^{2} \times \mathbb{S}^{1}$. Then $P_{W}$ is injective on $L_{0}^{p}\left(\mathbb{R}^{2}\right)$ for $p \geq 2$.

Injectivity 1 is a global injectivity for $d \geq 3$. Injectivity 2 is a local injectivity for $d=2$. Injectivity 3 is a global injectivity for $d=2$ for the rotation invariant case. Injectivity 4 is a global injectivity for $d=2$ for the real-analytic case.

The results of Injectivity 1 and Injectivity 2 remain valid with $C^{\alpha}, \alpha>1$, in place of $C^{2}$ in the assumptions on $W$; see [I16].

Injectivity 1 follows from Injectivity 2 in the framework of the layer-by-layer reconstruction approach. See [Fi86], [N02a], [I16] and references therein in connection with the layer-by-layer reconstruction approach for weighted and non-abelian ray transforms in dimension $d \geq 3$.

The work [B93] gives a counterexample to Injectivity 4 for $P_{W}$ in $C_{0}^{\infty}\left(\mathbb{R}^{2}\right)$ for the case when the assumption that $W$ is real-analytic is relaxed to the assumption that $W$ is infinitely smooth, where $C_{0}^{\infty}$ denotes infinitely smooth compactly supported functions.

In somewhat similar way with [B93], in the present work we obtain counterexamples to Injectivity 1, Injectivity 2 and Injectivity 3 for the case when the regularity of $W$ is slightly relaxed. In particular, by these counterexamples we continue related studies of [MQ85], [B93] and [GN18].

More precisely, in the present work we construct $W$ and $f$ such that

$$
P_{W} f \equiv 0 \quad \text { on } T \mathbb{S}^{d-1}, d \geq 2,
$$

where $W$ satisfies (1.4), $W$ is rotation-invariant (i.e., satisfies (1.9)),

$W$ is infinitely smooth almost everywhere on $\mathbb{R}^{d} \times \mathbb{S}^{d-1}$ and

$$
W \in C^{\alpha}\left(\mathbb{R}^{d} \times \mathbb{S}^{d-1}\right) \text {, at least, for any } \alpha \in\left(0, \alpha_{0}\right) \text {, where } \alpha_{0}=1 / 16 \text {; }
$$


$f$ is a non-zero spherically symmetric infinitely smooth and

see Theorem 1 of Section 3.

These $W$ and $f$ directly give the aforementioned counterexamples to Injectivity 1 and Injectivity 3 .

Our counterexample to Injectivity 1 is of particular interest (and is rather surprising) in view of the fact that the problem of finding $f$ on $\mathbb{R}^{d}$ from $P_{W} f$ on $T \mathbb{S}^{d-1}$ for known $W$ is strongly overdetermined for $d \geq 3$. Indeed,

$$
\begin{aligned}
& \operatorname{dim} \mathbb{R}^{d}=d, \quad \operatorname{dim} T \mathbb{S}^{d-1}=2 d-2, \\
& d<2 d-2 \quad \text { for } d \geq 3 .
\end{aligned}
$$

This counterexample to Injectivity 1 is also rather surprising in view of the aforementioned layer-by-layer reconstruction approach in dimension $d \geq 3$.

Our counterexample to Injectivity 3 is considerably stronger than the preceeding counterexample of [MQ85], where $W$ is not yet continuous and is not yet strictly positive (i.e., is not yet separated from zero by a positive constant).

Using our $W$ and $f$ of (1.11), (1.12) for $d=3$ we also obtain the aforementioned counterexample to Injectivity 2; see Corollary 1 of Section 3.

Finally, in the present work we also give examples of $W$ satisfying (1.4) such that dim ker $P_{W} \geq n$ in $C_{0}^{\infty}\left(\mathbb{R}^{d}\right)$ for arbitrary $n \in \mathbb{N} \cup\{\infty\}$, where $W \in C^{\infty}\left(\mathbb{R}^{2} \times \mathbb{S}^{1}\right)$ for $d=2$ and $W$ satisfy (1.11) for $d \geq 3$; see Theorem 2 of Section 3. To our knowledge, examples of $W$ satisfying (1.4), where $\operatorname{dim} \operatorname{ker} P_{W} \geq n$ (for example in $L_{0}^{2}\left(\mathbb{R}^{d}\right)$ ) were not yet given in the literature even for $n=1$ in dimension $d \geq 3$ and even for $n=2$ in dimension $d=2$.

In the present work we adopt and develop considerations of the famous work [B93] and of our very recent work [GN18].

In Section 2 we give some preliminaries and notations.

Main results are presented in detail in Sections 3.

Related proofs are given in Sections 4-9.

\section{Some preliminaries}

Notations Let

$$
\begin{aligned}
& \Omega=\mathbb{R}^{d} \times \mathbb{S}^{d-1}, \\
& r(x, \theta)=|x-(x \theta) \theta|, \quad(x, \theta) \in \Omega, \\
& \Omega_{0}(\delta)=\{(x, \theta) \in \Omega: r(x, \theta)>\delta\},
\end{aligned}
$$




$$
\begin{aligned}
& \Omega_{1}(\delta)=\Omega \backslash \Omega_{0}(\delta)=\{(x, \theta) \in \Omega: r(x, \theta) \leq \delta\}, \quad \delta>0, \\
& \Omega(\Lambda)=\left\{(x, \theta) \in \mathbb{R}^{d} \times \mathbb{S}^{d-1}: r(x, \theta) \in \Lambda\right\}, \quad \Lambda \subset[0,+\infty), \\
& T_{0}(\delta)=\left\{(x, \theta) \in T \mathbb{S}^{d-1}:|x|>\delta\right\}, \\
& T_{1}(\delta)=\left\{(x, \theta) \in T \mathbb{S}^{d-1}:|x| \leq \delta\right\}, \quad \delta>0, \\
& T(\Lambda)=\left\{(x, \theta) \in T \mathbb{S}^{d-1}:|x| \in \Lambda\right\}, \quad \Lambda \subset[0,+\infty), \\
& \mathcal{J}_{r, \varepsilon}=(r-\varepsilon, r+\varepsilon) \cap[0,+\infty), \quad r \in[0,+\infty), \varepsilon>0 .
\end{aligned}
$$

The set $T_{0}(\delta)$ in (2.6) is considered as the set of all rays in $\mathbb{R}^{d}$ which are located at distance greater than $\delta$ from the origin.

The set $T_{1}(\delta)$ in $(2.7)$ is considered as the set of all rays in $\mathbb{R}^{d}$ which are located at distance less or equal than $\delta$ from the origin.

We also consider the projection

$$
\begin{aligned}
& \pi: \Omega \longrightarrow T \mathbb{S}^{d-1}, \\
& \pi(x, \theta)=\left(\pi_{\theta} x, \theta\right), \quad(x, \theta) \in \Omega, \\
& \pi_{\theta} x=x-(x \theta) \theta .
\end{aligned}
$$

In addition, $r(x, \theta)$ of $(2.2)$ is the distance from the origin $\{0\} \in \mathbb{R}^{d}$ to the ray $\gamma=\gamma(\pi(x, \theta))$ (i.e., $\left.r(x, \theta)=\left|\pi_{\theta} x\right|\right)$. The rays will be also denoted by

$$
\gamma=\gamma(x, \theta) \stackrel{\text { def }}{=} \gamma(\pi(x, \theta)), \quad(x, \theta) \in \Omega .
$$

We also consider

$$
P_{W} f(x, \theta)=P_{W} f(\pi(x, \theta)) \quad \text { for }(x, \theta) \in \Omega .
$$

We also define

$$
\begin{aligned}
& B\left(x_{0}, \delta\right)=\left\{x \in \mathbb{R}^{d}:\left|x-x_{0}\right|<\delta\right\}, \\
& \bar{B}\left(x_{0}, \delta\right)=\left\{x \in \mathbb{R}^{d}:\left|x-x_{0}\right| \leq \delta\right\}, \quad x_{0} \in \mathbb{R}^{d}, \delta>0, \\
& B=B(0,1), \quad \bar{B}=\bar{B}(0,1) .
\end{aligned}
$$

For a function $f$ on $\mathbb{R}^{d}$ we denote its restriction to a subset $\Sigma \subset \mathbb{R}^{d}$ by $\left.f\right|_{\Sigma}$.

By $C_{0}, C_{0}^{\infty}$ we denote continuous compactly supported and infinitely smooth compactly supported functions, respectively.

By $C^{\alpha}(Y), \alpha \in(0,1)$, we denote the space of $\alpha$-Hölder functions on $Y$ with the norm:

$$
\begin{aligned}
& \|u\|_{C^{\alpha}(Y)}=\|u\|_{C(Y)}+\|u\|_{C^{\alpha}(Y)}^{\prime}, \\
& \|u\|_{C^{\alpha}(Y)}^{\prime}=\sup _{\substack{y_{1}, y_{2} \in Y \\
\left|y_{1}-y_{2}\right| \leq 1}} \frac{\left|u\left(y_{1}\right)-u\left(y_{2}\right)\right|}{\left|y_{1}-y_{2}\right|^{\alpha}},
\end{aligned}
$$

where $\|u\|_{C(Y)}$ denotes the supremum of $|u|$ on $Y$. 
Rotation invariance Using formula (1.6), for positive and continous $W$, property (1.9) can be rewritten in the following equivalent form:

$$
W(x, \theta)=U(|x-(x \theta) \theta|, x \theta), \quad x \in \mathbb{R}^{d}, \theta \in \mathbb{S}^{d-1},
$$

for some positive and continuous $U$ such that

$$
U(r, s)=U(-r, s)=U(r,-s), \quad r \in \mathbb{R}, s \in \mathbb{R} .
$$

In addition, symmetries (2.18), (2.19) of $W$ can be also written as

$$
\begin{aligned}
& W(x, \theta)=\widetilde{U}(|x|, x \theta), \quad(x, \theta) \in \Omega, \\
& \widetilde{U}(r, s)=\widetilde{U}(-r, s)=\widetilde{U}(r,-s), \quad r \in \mathbb{R}, s \in \mathbb{R} .
\end{aligned}
$$

where $\widetilde{U}$ is positive and continuous on $\mathbb{R} \times \mathbb{R}$. Using the formula $|x|^{2}=|x \theta|^{2}+r^{2}(x, \theta)$, one can see that symmetries (2.18), (2.19) and symmetries (2.20), (2.21) of $W$ are equivalent.

Partition of unity We recall the following classical result (see, e.g., Theorem 5.6 in $[\mathrm{M} 92])$ :

Let $\mathcal{M}$ be a $C^{\infty}$-manifold, which is Hausdorff and has a countable base. Let also $\left\{U_{i}\right\}_{i=1}^{\infty}$ be an open locally-finite cover of $\mathcal{M}$.

Then there exists a $C^{\infty}$-smooth locally-finite partition of unity $\left\{\psi_{i}\right\}_{i=1}^{\infty}$ on $\mathcal{M}$, such that

$$
\operatorname{supp} \psi_{i} \subset U_{i}
$$

In particular, any open interval $(a, b) \subset \mathbb{R}$ and $\Omega$ satisfy the conditions for $\mathcal{M}$ of this statement. It will be used in Subsection 3.1.

\section{Main results}

Theorem 1. There exist a weight $W$ satisfying (1.4) and a non-zero function $f \in C_{0}^{\infty}\left(\mathbb{R}^{d}\right), d \geq 2$, such that

$$
P_{W} f \equiv 0 \quad \text { on } T \mathbb{S}^{d-1},
$$

where $P_{W}$ is defined in (1.1). In addition, $W$ is rotation invariant, i.e., satisfies (2.18), and $f$ is spherically symmetric with supp $f \subseteq \bar{B}$. Moreover,

$$
\begin{aligned}
& W \in C^{\infty}(\Omega \backslash \Omega(1)), \\
& W \in C^{\alpha}\left(\mathbb{R}^{d} \times \mathbb{S}^{d-1}\right) \quad \text { for any } \alpha \in\left(0, \alpha_{0}\right), \alpha_{0}=1 / 16,
\end{aligned}
$$




$$
\begin{aligned}
& W \geq 1 / 2 \quad \text { on } \Omega \text { and } W \equiv 1 \text { on } \Omega([1,+\infty)) \\
& W(x, \theta) \equiv 1 \quad \text { for }|x| \geq R>1, \theta \in \mathbb{S}^{d-1}
\end{aligned}
$$

where $\Omega, \Omega(1), \Omega([1,+\infty))$ are defined by $(2.1),(2.5), R$ is a constant.

The construction of $W$ and $f$ proving Theorem 1 is presented below in Subsections 3.1, 3.2. In addition, this construction consists of its version in dimension $d=2$ (see Subsection 3.1) and its subsequent extension to the case of $d \geq 3$ (see Subsection 3.2).

Theorem 1 directly gives counterexamples to Injectivity 1 and Injectivity 3 of Introduction. Theorem 1 also implies the following counterexample to Injectivity 2 of Introduction:

Corollary 1. For any $\alpha \in(0,1 / 16)$ there is $N>0$ such that for any $\delta>0$ there are $W_{\delta}, f_{\delta}$ satisfying

$$
\begin{aligned}
& W_{\delta} \geq 1 / 2, \quad W_{\delta} \in C^{\alpha}\left(\mathbb{R}^{2} \times \mathbb{S}^{1}\right), \quad\left\|W_{\delta}\right\|_{C^{\alpha}\left(\mathbb{R}^{2} \times \mathbb{S}^{1}\right)} \leq N \\
& f_{\delta} \in C^{\infty}\left(\mathbb{R}^{2}\right), \quad f_{\delta} \not \equiv 0, \quad \operatorname{supp} f_{\delta} \subseteq \bar{B}(0, \delta), \\
& P_{W_{\delta}} f_{\delta} \equiv 0 \quad \text { on } T \mathbb{S}^{1} .
\end{aligned}
$$

The construction of $W_{\delta}, f_{\delta}$ proving Corollary 1 is presented in Subsection 5.1.

Theorem 2. For any $n \in \mathbb{N} \cup\{\infty\}$ there exists a weight $W_{n}$ satisfying (1.4) such that

$$
\operatorname{dim} \operatorname{ker} P_{W_{n}} \geq n \quad \text { in } C_{0}^{\infty}\left(\mathbb{R}^{d}\right), d \geq 2,
$$

where $P_{W}$ is defined in (1.1). Moreover,

$$
\begin{aligned}
& W_{n} \in C^{\infty}\left(\mathbb{R}^{2} \times \mathbb{S}^{1}\right) \quad \text { for } d=2, \\
& W_{n} \quad \text { is infinitely smooth almost everywhere on } \mathbb{R}^{d} \times \mathbb{S}^{d-1} \text { and } \\
& W_{n} \in C^{\alpha}\left(\mathbb{R}^{d} \times \mathbb{S}^{d-1}\right), \alpha \in(0,1 / 16) \text { for } d \geq 3, \\
& W_{n}(x, \theta) \equiv 1 \quad \text { for }|x| \geq R>1, \theta \in \mathbb{S}^{d-1} \text { for } n \in \mathbb{N}, d \geq 2,
\end{aligned}
$$

where $R$ is a constant.

The construction of $W_{n}$ proving Theorem 2 is presented in Section 4. In this construction we proceed from Theorem 1 of the present work for $d \geq 3$ and from the result of [B93] for $d=2$. In addition, for this construction it is essential that $n<+\infty$ in $(3.12)$. 


\subsection{Construction of $f$ and $W$ for $d=2$}

In dimension $d=2$, the construction of $f$ and $W$ adopts and develops considerations of [B93] and [GN18]. In particular, we construct $f$, first, and then $W$ (in this construction we use notations of Section 2 for $d=2$ ). In addition, this construction is commented in Remarks $1-5$ below.

Construction of $f$ The function $f$ is constructed as follows:

$$
\begin{aligned}
& f=\sum_{k=1}^{\infty} \frac{f_{k}}{k !}, \\
& f_{k}(x)=\tilde{f}_{k}(|x|)=\Phi\left(2^{k}(1-|x|)\right) \cos \left(8^{k}|x|^{2}\right), x \in \mathbb{R}^{2}, \quad k \in \mathbb{N},
\end{aligned}
$$

for arbitrary $\Phi \in C^{\infty}(\mathbb{R})$ such that

$$
\begin{aligned}
& \operatorname{supp} \Phi=[4 / 5,6 / 5] \\
& 0<\Phi(t) \leq 1 \quad \text { for } t \in(4 / 5,6 / 5) \\
& \Phi(t)=1, \quad \text { for } t \in[9 / 10,11 / 10] \\
& \Phi \text { monotonously increases on }[4 / 5,9 / 10] \\
& \quad \text { and monotonously decreases on }[11 / 10,6 / 5] .
\end{aligned}
$$

Properties (3.15), (3.16) imply that functions $\tilde{f}_{k}$ (and functions $f_{k}$ ) in (3.14) have disjoint supports:

$$
\begin{aligned}
& \operatorname{supp} \tilde{f}_{i} \cap \operatorname{supp} \tilde{f}_{j}=\varnothing \quad \text { if } i \neq j, \\
& \operatorname{supp} \tilde{f}_{k}=\left[1-2^{-k}\left(\frac{6}{5}\right), 1-2^{-k}\left(\frac{4}{5}\right)\right], \quad i, j, k \in \mathbb{N} .
\end{aligned}
$$

This implies the convergence of series in (3.13) for every fixed $x \in \mathbb{R}^{2}$.

Lemma 1. Let $f$ be defined by (3.13)-(3.17). Then $f$ is spherically symmetric, $f \in C_{0}^{\infty}\left(\mathbb{R}^{2}\right)$ and $\operatorname{supp} f \subseteq \bar{B}$. In addition, if $\gamma \in T \mathbb{S}^{1}, \gamma \cap B \neq \varnothing$, then $\left.f\right|_{\gamma} \neq 0$ and $\left.f\right|_{\gamma}$ has non-constant sign.

Lemma 1 is similar to Lemma 1 of [GN18] and it is, actually, proved in Section 4.1 of [GN18].

Remark 1. Formulas (3.13)-(3.17) for $f$ are similar to the formulas for $f$ in [B93], where $P_{W}$ was considered in $\mathbb{R}^{2}$, and also to the formulas for $f$ in [GN18], where the weighted Radon transform $R_{W}$ along hyperplanes was considered in $\mathbb{R}^{3}$. The only difference between (3.13)-(3.17) and the related formulas in [GN18] is the dimension $d=2$ in (3.13)-(3.17) instead of $d=3$ in [GN18]. At the same time, the 
important difference between (3.13)-(3.17) and the related formulas in [B93] is that in formula (3.14) the factor $\cos \left(8^{k}|x|^{2}\right)$ depends only on $|x|$, whereas in [B93] the corresponding factor is $\cos \left(3^{k} \phi\right)$ which depends only on the angle $\phi$ in the polar coordinates in $\mathbb{R}^{2}$. In a similar way with [B93], [GN18], we use the property that the restriction of the function $\cos \left(8^{k}|x|^{2}\right)$ to an arbitrary ray $\gamma$ intersecting the open ball oscillates sufficiently fast (with change of the sign) for large $k$.

Construction of $W$ In our example $W$ is of the following form:

$$
\begin{aligned}
W(x, \theta) & =\phi_{1}(x)\left(\sum_{i=0}^{N} \xi_{i}(r(x, \theta)) W_{i}(x, \theta)\right)+\phi_{2}(x) \\
& =\phi_{1}(x)\left(\xi_{0}(r(x, \theta)) W_{0}(x, \theta)+\sum_{i=1}^{N} \xi_{i}(r(x, \theta)) W_{i}(x, \theta)\right)+\phi_{2}(x), \\
(x, \theta) & \in \Omega,
\end{aligned}
$$

where

$$
\phi_{1}=\phi_{1}(|x|), \quad \phi_{2}=\phi_{2}(|x|)
$$

is a $C^{\infty}$-smooth partition of unity on $\mathbb{R}^{2}$ such that,

$$
\begin{array}{ll}
\phi_{1} \equiv 0 & \text { for }|x| \geq R>1, \phi_{1} \equiv 1 \text { for }|x| \leq 1, \\
\phi_{2} \equiv 0 & \text { for }|x| \leq 1,
\end{array}
$$

From the result of Lemma 1 and from (3.20), (3.21) it follows that

$$
P_{W} f(x, \theta)=\xi_{0}(|x|) P_{W_{0}} f(x, \theta)+\sum_{i=1}^{N} \xi_{i}(|x|) P_{W_{i}} f(x, \theta), \quad(x, \theta) \in T \mathbb{S}^{1},
$$

where $W$ is given by (3.20). Here, we also used that $r(x, \theta)=|x|$ for $(x, \theta) \in T \mathbb{S}^{1}$.

From (3.20)-(3.24) it follows that $W$ of (3.20) satisfies the conditions (1.4), $(2.20),(2.21)$.

The weight $W_{0}$ is constructed in next paragraph and has the following properties:

(3.26) $W_{0}$ is bounded, continuous and rotation invariant on $\Omega(1 / 2,+\infty)$, 


$$
W_{0} \in C^{\infty}(\Omega((1 / 2,1) \cup(1,+\infty))) \text { and }
$$

$$
W_{0} \in C^{\alpha}(\Omega(1 / 2,+\infty)) \text { for } \alpha \in(0,1 / 16) \text {, }
$$

there exists $\delta_{0} \in(1 / 2,1)$ such that:

$$
\begin{aligned}
W_{0}(x, \theta) & \geq 1 / 2 \quad \text { if } r(x, \theta)>\delta_{0}, \\
W_{0}(x, \theta) & =1 \quad \text { if } r(x, \theta) \geq 1, \\
P_{W_{0}} f(x, \theta) & =0 \text { on } \Omega((1 / 2,+\infty)),
\end{aligned}
$$

where $P_{W_{0}}$ is defined according to (1.1) for $W=W_{0}, f$ is given by (3.13), (3.14).

In addition,

$$
\begin{aligned}
& \operatorname{supp} \xi_{0} \subset\left(-\infty,-\delta_{0}\right) \cup\left(\delta_{0},+\infty\right), \\
& \xi_{0}(s)=1 \text { for }|s| \geq 1,
\end{aligned}
$$

where $\delta_{0}$ is the number of $(3.28)$.

In particular, from (3.28), (3.30) it follows that

$$
W_{0}(x, \theta) \xi_{0}(r(x, \theta))>0 \quad \text { if } \xi_{0}(r(x, \theta))>0 .
$$

In addition,

$$
\begin{aligned}
& \xi_{i}(r(x, \theta)) W_{i}(x, \theta) \quad \text { are bounded, rotation invariant and } C^{\infty} \text { on } \Omega, \\
& W_{i}(x, \theta) \geq 1 / 2 \text { if } \xi_{i}(r(x, \theta)) \neq 0, \\
& P_{W_{i}} f(x, \theta)=0 \quad \text { on }(x, \theta) \in T \mathbb{S}^{1}, \text { such that } \xi_{i}(r(x, \theta)) \neq 0, \\
& \quad i=\overline{1, N},(x, \theta) \in \Omega .
\end{aligned}
$$

Weights $W_{1}, \ldots, W_{N}$ of $(3.20)$ and $\left\{\xi_{i}\right\}_{i=0}^{N}$ are constructed in Subsection 3.1.

Theorem 1 for $d=2$ follows from Lemma 1 and formulas (3.20)-(3.29), (3.32)-(3.35).

We point out that the construction of $W_{0}$ of (3.20) is substantially different from the construction of $W_{1}, \ldots, W_{N}$. The weight $W_{0}$ is defined for the rays $\gamma \in T \mathbb{S}^{1}$ which can be close to the boundary $\partial B$ of $B$ which results in restrictions on global smoothness of $W_{0}$.

Remark 2. The construction of $W$ summarized above in formulas (3.20)-(3.35) arises in the framework of finding $W$ such that

$$
P_{W} f \equiv 0 \quad \text { on } T \mathbb{S}^{1} \text { for } f \text { defined in }(3.13)-(3.18),
$$

under the condition that $W$ is strictly positive, sufficiently regular and rotation invariant (see formulas (1.4), (2.18), (2.19)). In addition, the weights $W_{i}, i=0, \ldots, N$, in (3.20) are constructed in a such a way that

$$
P_{W_{i}} f=0 \quad \text { on } V_{i}, i=0, \ldots, N,
$$


under the condition that $W_{i}=W_{i}(x, \gamma)$ are strictly positive, sufficiently regular and rotation invariant for $x \in \gamma, \gamma \in V_{i} \subset T \mathbb{S}^{1}, i=0, \ldots, N$, where

$$
\begin{aligned}
& \left\{V_{i}\right\}_{i=0}^{N} \text { is an open cover of } T \mathbb{S}^{1} \text { and } V_{0}=T_{0}\left(\delta_{0}\right), \\
& V_{i}=T\left(\Lambda_{i}\right) \text { for some open } \Lambda_{i} \subset \mathbb{R}, i=0, \ldots, N,
\end{aligned}
$$

where $T_{0}$ is defined in (2.6), $\delta_{0}$ is the number of (3.28), $T(\Lambda)$ is defined in (2.8). In addition, the functions $\xi_{i}, i=0, \ldots, N$, in (3.20) can be interpreted as a partition of unity on $T \mathbb{S}^{1}$ subordinated to the open cover $\left\{V_{i}\right\}_{i=0}^{N}$. The aforementioned construction of $W$ is a two-dimensional analog of the construction developed in [GN18], where the weighted Radon transform $R_{W}$ along hyperplanes was considered in $\mathbb{R}^{3}$. At the same time, the construction of $W$ of the present work is similar to the construction in [B93] with the important difference that in the present work $f$ is spherically symmetric and $W, W_{i}, i=0, \ldots, N$, are rotation invariant.

Construction of $W_{0}$ Let $\left\{\psi_{k}\right\}_{k=1}^{\infty}$ be a $C^{\infty}$ partition of unity on $(1 / 2,1)$ such that

$$
\operatorname{supp} \psi_{k} \subset\left(1-2^{-k+1}, 1-2^{-k-1}\right), \quad k \in \mathbb{N},
$$

first derivatives $\psi_{k}^{\prime}$ satisfy the bounds: $\sup \left|\psi_{k}^{\prime}\right| \leq C 2^{k}$,

where $C$ is a positive constant. Actually, functions $\left\{\psi_{k}\right\}_{k=1}^{\infty}$ satisfying (3.40), (3.41) were used in considerations of [B93].

Note that

$$
1-2^{-(k-2)-1}<1-2^{-k}(6 / 5), \quad k \geq 3 .
$$

Therefore,

(3.43) for all $s_{0}, t_{0} \in \mathbb{R}, s_{0} \in \operatorname{supp} \psi_{k-2}, t_{0} \in \operatorname{supp} \Phi\left(2^{k}(1-t)\right) \Longrightarrow s_{0}<t_{0}, \quad k \geq 3$.

Weight $W_{0}$ is defined by the following formulas

$$
\begin{aligned}
& W_{0}(x, \theta)= \begin{cases}1-G(x, \theta) \sum_{k=3}^{\infty} k ! f_{k}(x) \frac{\psi_{k-2}(r(x, \theta))}{H_{k}(x, \theta)}, & 1 / 2<r(x, \theta)<1, \\
1, & r(x, \theta) \geq 1,\end{cases} \\
& G(x, \theta)=\int_{\gamma(x, \theta)} f(y) d y, \quad H_{k}(x, \theta)=\int_{\gamma(x, \theta)} f_{k}^{2}(y) d y, \quad x \in \mathbb{R}^{2}, \theta \in \mathbb{S}^{1},
\end{aligned}
$$

where $f, f_{k}$ are defined in (3.13), (3.14), respectively, rays $\gamma(x, \theta)$ are given by (2.13).

Formula (3.44) implies that $W_{0}$ is defined on $\Omega_{0}(1 / 2) \subset \Omega$. 
Due to $(3.14)-(3.17),(3.40),(3.43)$, in $(3.45)$ we have that

$$
\begin{aligned}
& H_{k}(x, \theta) \neq 0 \text { if } \psi_{k-2}(r(x, \theta)) \neq 0,(x, \theta) \in \Omega, \\
& \frac{\psi_{k-2}(r(x, \theta))}{H_{k}(x, \theta)} \in C^{\infty}(\Omega(1 / 2,1)),
\end{aligned}
$$

where $r(x, \theta)$ is defined in $(2.2), \Omega, \Omega(\cdot)$ are defined in $(2.1),(2.5), d=2$.

Also, for any fixed $(x, \theta) \in \Omega, 1 / 2<r(x, \theta)$, the series in the right hand-side of (3.44) has only a finite number of non-zero terms (in fact, no more than two) and, hence, the weight $W_{0}$ is well-defined.

By the spherical symmetry of $f$, functions $G, H_{k}$ in (3.44) are of the type (2.18) (and (2.20)). Therefore, $W_{0}$ is rotation invariant (in the sense of (2.18) and (2.20)).

Actually, formula (3.29) follows from (3.13), (3.14), (3.44), (3.45) (see Subsection 6.2 for details).

Using the construction of $W_{0}$ and the assumption that $r(x, \theta)>1 / 2$ one can see that $W_{0}$ is $C^{\infty}$ on its domain of definition, possibly, except points with $r(x, \theta)=1$.

Note also that due to (3.13), (3.14), the functions $f_{k}, G, H_{k}$, used in (3.44), (3.45) can be considered as functions of one-dimensional arguments.

Formulas (3.26)-(3.28) are proved in Subsection 6.1.

Remark 3. Formulas (3.44), (3.45) given above for the weight $W_{0}$ are considered for the rays from $T_{0}\left(\delta_{0}\right)$ (mentioned in Remark 2) and, in particular, for rays close to the tangent rays to $\partial B$. These formulas are direct two-dimensional analogs of the related formulas in [GN18]. At the same time, formulas (3.44), (3.45) are similar to the related formulas in [B93] with the important difference that $f, f_{k}$ are spherically symmetric in the present work and, as a corollary, $W_{0}$ is rotation invariant. Also, in a similar way with [B93], [GN18], in the present work we show that $G(x, \theta)$ tends to zero sufficiently fast as $r(x, \theta) \rightarrow 1$. This is a very essential point for continuity of $W_{0}$ and it is given in Lemma 3 of Subsection 6.1.

Construction of $W_{1}, \ldots, W_{N}$ and $\xi_{0}, \ldots, \xi_{N}$

Lemma 2. Let $f \in C_{0}^{\infty}\left(\mathbb{R}^{2}\right)$ be spherically symmetric, $\left(x_{0}, \theta_{0}\right) \in T \mathbb{S}^{1}$, $\left.f\right|_{\gamma\left(x_{0}, \theta_{0}\right)} \neq \equiv 0$ and $\left.f\right|_{\gamma\left(x_{0}, \theta_{0}\right)}$ changes the sign. Then there exist $\varepsilon_{0}>0$ and weight $W_{\left(x_{0}, \theta_{0}\right), \varepsilon_{0}}$ such that

$$
\begin{aligned}
& P_{W_{\left(x_{0}, \theta_{0}\right), \varepsilon_{0}}} f=0 \text { on } \Omega\left(\mathcal{J}_{r\left(x_{0}, \theta_{0}\right), \varepsilon_{0}}\right), \\
& W_{\left(x_{0}, \theta_{0}\right), \varepsilon_{0}} \text { is bounded, infinitely smooth, } \\
& \quad \text { strictly positive and rotation invariant on } \Omega\left(\mathcal{J}_{r\left(x_{0}, \theta_{0}\right), \varepsilon_{0}}\right),
\end{aligned}
$$

where $\Omega\left(\mathcal{J}_{r, \varepsilon_{0}}\right), \mathcal{J}_{r, \varepsilon_{0}}$ are defined in (2.5) and (2.9), respectively. 
Lemma 2 is proved in Section 7. This lemma is a two-dimensional analog of the related lemma in [GN18].

Remark 4. In Lemma 2 the construction of $W_{\left(x_{0}, \theta_{0}\right), \varepsilon_{0}}$ arises from

1. finding strictly positive and regular weight $W_{\left(x_{0}, \theta_{0}\right), \varepsilon}$ on the rays $\gamma=\gamma(x, \theta)$ with fixed $\theta=\theta_{0}$, where $r\left(x, \theta_{0}\right) \in \mathcal{J}_{r\left(x_{0}, \theta_{0}\right), \varepsilon}$ for some $\varepsilon>0$, such that (3.48) holds for $\theta=\theta_{0}$ and under the condition that

$$
W_{\left(x_{0}, \theta_{0}\right), \varepsilon}(y, \gamma)=W_{\left(x_{0}, \theta_{0}\right), \varepsilon}\left(\left|y \theta_{0}\right|, \gamma\right), \quad y \in \gamma=\gamma\left(x, \theta_{0}\right), r\left(x, \theta_{0}\right) \in \mathcal{J}_{r\left(x_{0}, \theta_{0}\right), \varepsilon}
$$

2. extending $W_{r\left(x_{0}, \theta_{0}\right), \varepsilon}$ to all rays $\gamma=\gamma(x, \theta), r(x, \theta) \in \mathcal{J}_{r\left(x_{0}, \theta_{0}\right), \varepsilon}, \quad \theta \in \mathbb{S}^{1}$, via formula (1.9).

We recall that $r(x, \theta)$ is defined in $(2.2)$.

Let $f$ be the function of $(3.13),(3.14)$. Then, using Lemmas 1,2 one can see that

$$
\begin{gathered}
\text { for all } \delta \in(0,1) \text { there exist }\left\{J_{i}=\mathcal{J}_{r_{i}, \varepsilon_{i}}, W_{i}=W_{\left(x_{i}, \theta_{i}\right), \varepsilon_{i}}\right\}_{i=1}^{N} \\
\text { such that } J_{i}, i=\overline{1, N} \text {, is an open cover of }[0, \delta] \text { in } \mathbb{R}, \\
\text { and } W_{i} \text { satisfy (3.48) and (3.49) on } \Omega\left(J_{i}\right) \text {, respectively. }
\end{gathered}
$$

Actually, we consider (3.51) for the case of $\delta=\delta_{0}$ of (3.28).

Note that in this case $\left\{\Omega\left(J_{i}\right)\right\}_{i=1}^{N}$ for $J_{i}$ of (3.51) is the open cover of $\Omega_{1}\left(\delta_{0}\right)$.

To the set $\Omega_{0}\left(\delta_{0}\right)$ we associate the open set

$$
J_{0}=\left(\delta_{0},+\infty\right) \subset \mathbb{R} .
$$

Therefore, the collection of intervals $\left\{ \pm J_{i}, i=\overline{0, N}\right\}$ is an open cover of $\mathbb{R}$, where $-J_{i}$ is the symmetrical reflection of $J_{i}$ with respect to $\{0\} \in \mathbb{R}$.

We construct the partition of unity $\left\{\xi_{i}\right\}_{i=0}^{N}$ as follows:

$$
\begin{aligned}
& \xi_{i}(s)=\xi_{i}(|s|)=\frac{1}{2}\left(\tilde{\xi}_{i}(s)+\tilde{\xi}_{i}(-s)\right), \quad s \in \mathbb{R}, \\
& \operatorname{supp} \xi_{i} \subset J_{i} \cup\left(-J_{i}\right), \quad i=\overline{0, N},
\end{aligned}
$$

where $\left\{\tilde{\xi}_{i}\right\}_{i=0}^{N}$ is a partition of unity for the open cover $\left\{J_{i} \cup\left(-J_{i}\right)\right\}_{i=0}^{N}$ (see Section 2, Partition of unity, for $\left.U_{i}=J_{i} \cup\left(-J_{i}\right)\right)$.

Properties (3.30), (3.54) follow from (2.22) for $\left\{\tilde{\xi}_{i}\right\}_{i=0}^{N}$ with $U_{i}=J_{i} \cup\left(-J_{i}\right)$, the symmetry of $J_{i} \cup\left(-J_{i}\right), i=\overline{1, N}$, choice of $J_{0}$ in (3.52) and from (3.53).

In turn, (3.31) follows from (3.52) and the construction of $J_{i}, i=\overline{1, N}$, from (3.51) (see the proof of Lemma 2 and properties (3.51) in Section 7 for details).

Properties (3.33)-(3.35) follow from (3.51) for $\delta=\delta_{0}$ and from (3.52)-(3.54).

This completes the description of $W_{1}, \ldots, W_{N}$ and $\left\{\xi_{i}\right\}_{i=0}^{N}$. 
Remark 5. We have that $J_{i}=\Lambda_{i}, i=1, \ldots, N$, where $\Lambda_{i}$ are the intervals in formula (3.39) of Remark 2 and $J_{i}$ are the intervals considered in (3.51), (3.52).

\subsection{Construction of $W$ and $f$ for $d \geq 3$}

Consider $f$ and $W$ of Theorem 1, for $d=2$, constructed in Subsection 3.1. For these $f$ and $W$ consider $\tilde{f}$ and $\widetilde{U}$ such that

$$
f(x)=\tilde{f}(|x|), \quad W(x, \theta)=\widetilde{U}(|x|,|x \theta|), x \in \mathbb{R}^{2}, \theta \in \mathbb{S}^{1} .
$$

Proposition 1. Let $W$ and $f$, for $d \geq 3$, be defined as

$$
\begin{aligned}
& W(x, \theta)=\tilde{U}(|x|,|x \theta|), \quad(x, \theta) \in \mathbb{R}^{d} \times \mathbb{S}^{d-1}, \\
& f(x)=\tilde{f}(|x|), \quad x \in \mathbb{R}^{d},
\end{aligned}
$$

where $\widetilde{U}, \tilde{f}$ are the functions of (3.55). Then

$$
P_{W} f \equiv 0 \quad \text { on } T \mathbb{S}^{d-1}
$$

In addition, weight $W$ satisfies properties (3.2)-(3.5), $f$ is spherically symmetric infinitely smooth and compactly supported on $\mathbb{R}^{d}, f \not \equiv$.

Proposition 1 is proved in Subsection 5.2.

This completes the proof of Theorem 1.

\section{Proof of Theorem 2}

\subsection{Proof for $d \geq 3$}

Let

(4.1) $W$ be the weight of Theorem 1 for $d \geq 3$,

(4.2) $\quad R$ be the number in (3.5) for $d \geq 3$,

$\left\{y_{i}\right\}_{i=1}^{\infty}$ be a sequence of vectors in $\mathbb{R}^{d}$ such that $y_{1}=0,\left|y_{i}-y_{j}\right|>2 R$

(4.3) for $i \neq j, i, j \in \mathbb{N}$,

$\left\{\bar{B}_{i}\right\}_{i=1}^{\infty}$ be the closed balls in $\mathbb{R}^{d}$ of radius $R$ centered at $y_{i}$

(see $(4.2),(4.3))$. 
The weight $W_{n}$ is defined as follows

$$
\begin{aligned}
& W_{n}(x, \theta)= \begin{cases}1 & \text { if } x \notin \bigcup_{i=1}^{n} \bar{B}_{i}, \\
W\left(x-y_{1}, \theta\right)=W(x, \theta) & \text { if } x \in \bar{B}_{1}, \\
W\left(x-y_{2}, \theta\right) & \text { if } x \in \bar{B}_{2}, \\
\ldots, & \\
W\left(x-y_{k}, \theta\right) & \text { if } x \in \bar{B}_{k}, \\
\cdots, & \text { if } x \in \bar{B}_{n}, \\
W\left(x-y_{n}, \theta\right) & \end{cases} \\
& \theta \in \mathbb{S}^{d-1}, \quad n \in \mathbb{N} \cup\{\infty\}, d \geq 3,
\end{aligned}
$$

where $W$ is defined in (4.1), $y_{i}$ and $\bar{B}_{i}$ are defined in (4.3), (4.4), respectively.

Properties (1.4), (3.11) and (3.12) for $W_{n}$, defined in (4.5), for $d \geq 3$, follow from $(3.2)-(3.5),(4.1),(4.2)$.

Let

$$
f_{1}(x) \stackrel{\text { def }}{=} f(x), \quad f_{2}(x) \stackrel{\text { def }}{=} f\left(x-y_{2}\right), \ldots, f_{n}(x) \stackrel{\text { def }}{=} f\left(x-y_{n}\right), x \in \mathbb{R}^{d}, d \geq 3,
$$

where $y_{i}$ are defined in (4.3) and

$$
f \text { is the function of Theorem } 1 \text { for } d \geq 3 \text {. }
$$

One can see that

$$
f_{i} \in C_{0}^{\infty}\left(\mathbb{R}^{d}\right), \quad d \geq 3, f_{i} \not \equiv 0, \operatorname{supp} f_{i} \subset \bar{B}_{i}, \bar{B}_{i} \cap \bar{B}_{j}=\varnothing \text { for } i \neq j,
$$

where $\bar{B}_{i}$ are defined in $(4.4), i=1, \ldots, n$.

The point is that

$$
P_{W_{n}} f_{i} \equiv 0 \quad \text { on } T \mathbb{S}^{d-1}, d \geq 3, i=1, \ldots, n,
$$

where $W_{n}$ is defined in (4.5), $f_{i}$ are defined in (4.6).

To prove (4.9) we use, in particular, the following general formula:

$$
\begin{aligned}
P_{W_{y}} f_{y}(x, \theta) & =\int_{\gamma(x, \theta)} W\left(y^{\prime}-y, \theta\right) f\left(y^{\prime}-y\right) d y^{\prime} \\
& =\int_{\gamma(x-y, \theta)} W\left(y^{\prime}, \theta\right) f\left(y^{\prime}\right) d y^{\prime}=P_{W} f(x-y, \theta), \quad x \in \mathbb{R}^{d}, \theta \in \mathbb{S}^{d-1},
\end{aligned}
$$




$$
W_{y}(x, \theta)=W(x-y, \theta), \quad f_{y}=f(x-y), x, y \in \mathbb{R}^{d}, \theta \in \mathbb{S}^{d-1} .
$$

where $W$ is an arbitrary weight satisfying (1.4), $f$ is a test-function, $\gamma(x, \theta)$ is defined according to (2.13).

Formula (4.9) follows from formula (3.1), definitions (4.5), (4.6), (4.7), properties (4.8) and from formulas (4.11), (4.12).

Formula (4.10) follows from definitions (4.6), (4.7) and properties (4.8).

This completes the proof of Theorem 2 for $d \geq 3$.

\subsection{Proof for $d=2$}

In [B93], there were constructed a weight $W$ and a function $f$ for $d=2$, such that:

$$
\begin{aligned}
& P_{W} f \equiv 0 \quad \text { on } T \mathbb{S}^{1}, \\
& W=\bar{W} \geq c>0, \quad W \in C^{\infty}\left(\mathbb{R}^{2} \times \mathbb{S}^{1}\right), \\
& f \in C_{0}^{\infty}\left(\mathbb{R}^{2}\right), \quad f \not \equiv 0, \operatorname{supp} f \subset \bar{B},
\end{aligned}
$$

where $c$ is a constant, $\bar{B}$ is defined in (2.16).

We define

$$
\widetilde{W}(x, \theta)=c^{-1} \phi_{1}(x) W(x, \theta)+\phi_{2}(x), \quad x \in \mathbb{R}^{2}, \theta \in \mathbb{S}^{1},
$$

where $W$ is the weight of (4.13), (4.14), $c$ is a constant of (4.14).

$$
\begin{aligned}
& \phi_{1}=\phi_{1}(x), \quad \phi_{2}=\phi_{2}(x) \text { is a } C^{\infty} \text {-smooth partition of unity on } \mathbb{R}^{2} \text { such that, } \\
& \phi_{1} \equiv 0 \text { for }|x| \geq R>1, \phi_{1} \equiv 1 \text { for }|x| \leq 1, \phi_{1} \geq 0 \text { on } \mathbb{R}^{2}, \\
& \phi_{2} \equiv 0 \text { for }|x| \leq 1, \phi_{2} \geq 0 \text { on } \mathbb{R}^{2},
\end{aligned}
$$

where $R$ is a constant.

From (4.13)-(4.17) it follows that

$$
\begin{aligned}
& P_{\widetilde{W}} f \equiv 0 \quad \text { on } T \mathbb{S}^{1}, \\
& \widetilde{W} \geq 1, \quad \widetilde{W} \in C^{\infty}\left(\mathbb{R}^{2} \times \mathbb{S}^{1}\right), \\
& \widetilde{W}(x, \theta) \equiv 1 \quad \text { for }|x| \geq R>1, \theta \in \mathbb{S}^{1} .
\end{aligned}
$$

The proof of Theorem 2 for $d=2$ proceeding from (4.15), (4.16), (4.18), (4.19) is completely similar to the proof of Theorem 2 for $d \geq 3$, proceeding from Theorem 1 .

Theorem 2 is proved. 


\section{Proofs of Corollary 1 and Proposition 1}

\subsection{Proof of Corollary 1}

Let

$$
\begin{aligned}
& X_{r}=\left\{x_{1} e_{1}+x_{2} e_{2}+r e_{3}:\left(x_{1}, x_{2}\right) \in \mathbb{R}^{2}\right\}, \quad 0 \leq r<1, \\
& S=X_{0} \cap \mathbb{S}^{2}=\left\{(\cos \phi, \sin \phi, 0) \in \mathbb{R}^{3}: \phi \in[0,2 \pi)\right\} \simeq \mathbb{S}^{1} .
\end{aligned}
$$

where $\left(e_{1}, e_{2}, e_{3}\right)$ is the standard orthonormal basis in $\mathbb{R}^{3}$.

Without loss of generality we assume that $0<\delta<1$. Choosing $r$ so that $\sqrt{1-\delta^{2}} \leq$ $r<1$, we have that the intersection of the three dimensional ball $B(0,1)$ with $X_{r}$ is the two-dimensional disk $B\left(0, \delta^{\prime}\right), \delta^{\prime} \leq \delta$ (with respect to the coordinates $\left(x_{1}, x_{2}\right)$ induced by basis $\left(e_{1}, e_{2}\right)$ on $\left.X_{r}\right)$.

We define $N, W_{\delta}$ on $\mathbb{R}^{2} \times \mathbb{S}^{1}$ and $f_{\delta}$ on $\mathbb{R}^{2}$ as follows:

$$
\begin{aligned}
& N=\|W\|_{C^{\alpha}\left(\mathbb{R}^{3} \times \mathbb{S}^{2}\right)}, \\
& W_{\delta}:=\left.W\right|_{X_{r} \times S}, \\
& f_{\delta}:=\left.f\right|_{X_{r}} \\
& \quad \text { for } r=\sqrt{1-\delta^{2}}
\end{aligned}
$$

where $W$ and $f$ are the functions of Theorem 1 for $d=3$.

Due to (3.2)-(3.4), (5.3), (5.4) we have that

$$
W_{\delta} \geq 1 / 2, \quad\left\|W_{\delta}\right\|_{C^{\alpha}\left(\mathbb{R}^{2} \times \mathbb{S}^{1}\right)} \leq N .
$$

Properties (5.6) imply (3.6).

In view of Lemma 1 for the function $f$ of Theorem 1 , we have that $f_{\delta}$ is spherically symmetric, $f_{\delta} \in C_{0}^{\infty}\left(B\left(0, \delta^{\prime}\right)\right), f_{\delta} \not \equiv$.

Using (3.1), (5.4), (5.5) one can see that (3.8) holds.

This completes the proof of Corollary 1.

\subsection{Proof of Proposition 1}

Let

$$
I(r)=\int_{\gamma_{r}} \tilde{U}(|y|, r) \tilde{f}(|y|) d y, \quad r \geq 0, \gamma_{r}=\gamma\left(r e_{2}, e_{1}\right)
$$

where $\gamma(x, \theta)$ is defined by $(1.3),\left(e_{1}, \ldots, e_{d}\right)$ is the standard basis in $\mathbb{R}^{d}$. 
Due to formula (3.1) of Theorem 1 for $d=2$ and formulas (3.55), (5.7) we have that

$$
I(r)=P_{W} f\left(r e_{2}, e_{1}\right)=0 \quad \text { for } r \geq 0 .
$$

Next, using (1.1), (3.55), (5.8) we have also that

(5.9) $P_{W} f(x, \theta)=\int_{\gamma(x, \theta)} \tilde{U}(|y|,|y-(y \theta) \theta|) \tilde{f}(|y|) d y=I(|x|)=0 \quad$ for $(x, \theta) \in T \mathbb{S}^{d-1}$,

where $\gamma(x, \theta)$ is defined in (1.3).

Formula (5.9) implies (3.58). Properties of $W$ and $f$ mentioned in Proposition 1 follow from properties (3.2)-(3.5) of $W$ and of $f$ of Theorem 1 for $d=2$.

This completes the proof of Proposition 1.

\section{Proofs of formulas $(3.26)-(3.29)$}

\subsection{Proof of formulas $(3.26)-(3.28)$}

Lemma 3. Let $W_{0}$ be defined by (3.44), (3.45). Then $W_{0}$ admits the following representation:

$$
\begin{aligned}
& W_{0}(x, \theta)=U_{0}(|x-(x \theta) \theta|, x \theta), \quad,(x, \theta) \in \Omega((1 / 2,+\infty)), \\
& U_{0}(r, s)= \begin{cases}1-\widetilde{G}(r) \sum_{k=3}^{\infty} k ! \tilde{f}_{k}\left(\left(s^{2}+r^{2}\right)^{1 / 2}\right) \frac{\psi_{k-2}(r)}{\widetilde{H}_{k}(r)}, & 1 / 2<r<1, \\
1, & r \geq 1,\end{cases} \\
& \widetilde{G}(r) \stackrel{\text { def }}{=} \int_{\gamma_{r}} \tilde{f}(|y|) d y, \quad \widetilde{H}_{k}(r) \stackrel{\text { def }}{=} \int_{\gamma_{r}} \tilde{f}_{k}^{2}(|y|) d y, \quad \tilde{f}=\sum_{k=1}^{\infty} \frac{\tilde{f}_{k}}{k !},
\end{aligned}
$$

where $\tilde{f}_{k}$ are defined by (3.14), $T(r)$ is defined by (2.8), $d=2$. In addition:

(6.4) $U_{0}$ is infinitely smooth on $\{(1 / 2,1) \cup(1,+\infty)\} \times \mathbb{R}$,

(6.5) $\quad U_{0}(r, s) \longrightarrow 1$ as $r \longrightarrow 1$ (uniformly in $s \in \mathbb{R}$ ),

(6.6) $\quad U_{0}(r, s)=1 \quad$ if $s^{2}+r^{2} \geq 1$,

$$
\begin{aligned}
& \left|1-U_{0}(r, s)\right| \leq C_{0}(1-r)^{1 / 2} \log _{2}^{4}\left(\frac{1}{1-r}\right), \\
& \quad \text { for } 1 / 2<r<1, s \in \mathbb{R}
\end{aligned}
$$




$$
\left|U_{0}(r, s)-U_{0}\left(r^{\prime}, s^{\prime}\right)\right| \leq C_{1}\left|s-s^{\prime}\right|^{\alpha}+C_{1}\left|r-r^{\prime}\right|^{\alpha},
$$

$$
\text { for } \alpha \in(0,1 / 16), r, r^{\prime}>1 / 2, s, s^{\prime} \in \mathbb{R},\left|r-r^{\prime}\right| \leq 1,\left|s-s^{\prime}\right| \leq 1
$$

where $C_{0}, C_{1}$ are positive constants depending on $\Phi$ of (3.15)-(3.17).

Lemma 3 is proved Section 8.

Lemma 3 implies (3.26)-(3.28) as follows.

The continuity and rotation invariancy of $W_{0}$ in (3.26) follow from (2.18), (2.19), (6.1), (6.8).

Due to $(3.40),(6.1),(6.2),(6.3)$ we have also that

$$
U_{0} \quad \text { admits a continuous extension to }[1 / 2,+\infty) \times \mathbb{R} \text {. }
$$

Properties (6.6), (6.9) imply the boundedness of $W_{0}$ on $\Omega_{0}(1 / 2)$, where $\Omega_{0}(\cdot)$ is defined in (2.3), $d=2$. This completes the proof of (3.26).

Formula (3.27) follows from $(6.1),(6.4),(6.8)$ and from the fact that $x \theta$, $|x-(x \theta) \theta|$ are infinitely smooth functions on $\Omega_{0}(1 / 2)$ and are Lipshitz in $(x, \theta)$ for $x \in \bar{B}(0, R), R>1$.

Formula (3.28) follows from (3.26), (6.1), (6.2), (6.5), (6.6).

This completes the proof of $(3.26)-(3.28)$.

\subsection{Proof of formula (3.29)}

From (1.1), (3.13)-(3.16), (3.40), (3.44), (3.45) it follows that:

$$
\begin{aligned}
P_{W_{0}} f(x, \theta) & =\int_{\gamma(x, \theta)} f(y) d y-G(x, \theta) \sum_{k=3}^{\infty} k ! \psi_{k-2}(r(x, \theta)) \frac{\int_{\gamma(x, \theta)} f(y) f_{k}(y) d y}{H_{k}(x, \theta)} \\
& =\int_{\gamma(x, \theta)} f(y) d y-\int_{\gamma(x, \theta)} f(y) d y \sum_{k=3}^{\infty} \psi_{k-2}(r(x, \theta)) \frac{\int_{\gamma(x, \theta)} f_{k}^{2}(y) d y}{\int_{\gamma(x, \theta)}^{2}(y) d y} \\
& =\int_{\gamma(x, \theta)} f(y) d y-\int_{\gamma(x, \theta)} f(y) d y \sum_{k=3}^{\infty} \psi_{k-2}(r(x, \theta))=0 \text { for }(x, \theta) \in \Omega_{0}(1 / 2),
\end{aligned}
$$

where $\gamma(x, \theta)$ is defined in (1.3), $\Omega_{0}(\cdot)$ is defined in $(2.3), d=2$.

Formula (3.29) is proved. 


\section{Proof of Lemma 2}

By $u \in \mathbb{R}$ we denote the coordinates on a fixed ray $\gamma(x, \theta),(x, \theta) \in \Omega, d=2$, taking into account the orientation, where $u=0$ at the point $x-(x \theta) \theta \in \gamma(x, \theta)$; see notation (2.13).

Using Lemma 1, one can see that

$$
\left.f\right|_{\gamma(x, \theta)} \in C_{0}^{\infty}(\mathbb{R}),\left.\quad f\right|_{\gamma(x, \theta)}(u)=\left.f\right|_{\gamma(x, \theta)}(|u|), u \in \mathbb{R} .
$$

Using (7.1) and the assumption that $\left.f\right|_{\gamma\left(x_{0}, \theta_{0}\right)}(u)$ changes the sign, one can see that there exists $\psi_{\left(x_{0}, \theta_{0}\right)}$ such that

$$
\begin{aligned}
& \psi_{\left(x_{0}, \theta_{0}\right)} \in C_{0}^{\infty}(\mathbb{R}), \quad \psi_{\left(x_{0}, \theta_{0}\right)} \geq 0, \quad \psi_{\left(x_{0}, \theta_{0}\right)}(u)=\psi_{\left(x_{0}, \theta_{0}\right)}(|u|), \quad u \in \mathbb{R}, \\
& \int_{\gamma\left(x_{0}, \theta_{0}\right)} f \psi_{\left(x_{0}, \theta_{0}\right)} d \sigma \neq 0,
\end{aligned}
$$

and if

$$
\int_{\gamma\left(x_{0}, \theta_{0}\right)} f d \sigma \neq 0
$$

then also

$$
\operatorname{sgn}\left(\int_{\gamma\left(x_{0}, \theta_{0}\right)} f d \sigma\right) \operatorname{sgn}\left(\int_{\gamma\left(x_{0}, \theta_{0}\right)} f \psi_{\left(x_{0}, \theta_{0}\right)} d \sigma\right)=-1,
$$

where $d \sigma=d u$ (i.e., $\sigma$ is the standard Euclidean measure on $\gamma(x, \theta)$ ).

Let

$$
W_{\left(x_{0}, \theta_{0}\right)}(x, \theta)=1-\psi_{\left(x_{0}, \theta_{0}\right)}(x \theta) \frac{\int_{\gamma(x, \theta)} f d \sigma}{\int_{\gamma(x, \theta)} f \psi_{\left(x_{0}, \theta_{0}\right)} d \sigma}, \quad x \in \mathbb{R}^{2}, \theta \in \mathbb{S}^{1},
$$

where $d \sigma=d u$, where $u$ is the coordinate on $\gamma(x, \theta)$.

Lemma 1 and property (7.2) imply that

$$
\begin{aligned}
& \int_{\gamma(x, \theta)} f d \sigma \text { and } \int_{\gamma(x, \theta)} f \psi_{\left(x_{0}, \theta_{0}\right)} d \sigma \text { depend only on } r(x, \theta), \\
& \text { where }(x, \theta) \in \Omega,
\end{aligned}
$$

where $r(x, \theta)$ is defined in $(2.2), \Omega$ is defined in $(2.1), d=2$.

From $(7.2),(7.6),(7.7)$ it follows that $W_{\left(x_{0}, \theta_{0}\right)}$ is rotation-invariant in the sense (2.18). 
Formulas (7.3), (7.6), (7.7), properties of $f$ of Lemma 1 and properties of $\psi_{\left(x_{0}, \theta_{0}\right)}$ of $(7.2)$ imply that

$$
\exists \varepsilon_{1}>0: \int_{\gamma(x, \theta)} f \psi_{\left(x_{0}, \theta_{0}\right)} d \sigma \neq 0 \quad \text { for }(x, \theta) \in \Omega\left(\mathcal{J}_{r\left(x_{0}, \theta_{0}\right), \varepsilon_{1}}\right),
$$

where sets $\Omega\left(\mathcal{J}_{s, \varepsilon}\right), \mathcal{J}_{s, \varepsilon}$ are defined in (2.5), (2.9), respectively.

In addition, using properties of $f$ of Lemma 1 and also using (3.13), (3.19), (7.2), (7.6), (7.8), one can see that

$$
W_{\left(x_{0}, \theta_{0}\right)} \in C^{\infty}\left(\Omega\left(\mathcal{J}_{r\left(x_{0}, \theta_{0}\right), \varepsilon_{1}}\right)\right) .
$$

In addition, from (7.1)-(7.7) it follows that

$$
\begin{aligned}
& \text { if } r(x, \theta)=r\left(x_{0}, \theta_{0}\right) \text { then } W_{\left(x_{0}, \theta_{0}\right)}(x, \theta)=1-\psi_{\left(x_{0}, \theta_{0}\right)}(x \theta) \frac{\iint_{\gamma\left(x_{0}, \theta_{0}\right)} f d \sigma}{\int \psi_{\left(x_{0}, \theta_{0}\right)} f \psi_{\left(x_{0}, \theta_{0}\right)} d \sigma} \\
& =1-\psi_{\left(x_{0}, \theta_{0}\right)}(x \theta) \frac{\int_{\gamma\left(x_{0}, \theta_{0}\right)} f d \sigma}{\int_{\gamma\left(x_{0}, \theta_{0}\right)} f \psi_{\left(x_{0}, \theta_{0}\right)} d \sigma} \geq 1 \text {, }
\end{aligned}
$$

where $r(x, \theta)$ is defined in $(2.2), d=2$.

From properties of $f$ of Lemma 1 , properties of $\psi_{\left(x_{0}, \theta_{0}\right)}$ of $(7.2)$ and from formulas (7.6), (7.8), (7.9), (7.10) it follows that

$$
\exists \varepsilon_{0}>0\left(\varepsilon_{0}<\varepsilon_{1}\right): W_{\left(x_{0}, \theta_{0}\right)}(x, \theta) \geq 1 / 2 \quad \text { for }(x, \theta) \in \Omega\left(\mathcal{J}_{r\left(x_{0}, \theta_{0}\right), \varepsilon_{0}}\right) .
$$

Let

$$
W_{\left(x_{0}, \theta_{0}\right), \varepsilon_{0}}:=W_{\left(x_{0}, \theta_{0}\right)} \quad \text { for }(x, \theta) \in \Omega\left(\mathcal{J}_{r\left(x_{0}, \theta_{0}\right), \varepsilon_{0}}\right),
$$

where $W_{\left(x_{0}, \theta_{0}\right)}$ is defined in (7.6).

Properties (7.7), (7.9), (7.11) imply (3.49) for $W_{\left(x_{0}, \theta_{0}\right), \varepsilon_{0}}$ of (7.12).

Using (1.1), (7.6), (7.8), (7.12) one can see that

$$
\begin{aligned}
& P_{W_{\left(x_{0}, \theta_{0}\right), \varepsilon_{0}}} f(x, \theta)=\int_{\gamma(x, \theta)} W_{\left(x_{0}, \theta_{0}\right)}(\cdot, \theta) f d \sigma \\
&=\int_{\gamma(x, \theta)} f d \sigma-\frac{\int_{\gamma(x, \theta)} f d \sigma}{\int_{\gamma(x, \theta)} f \psi_{\left(x_{0}, \theta_{0}\right)} d \sigma} \int_{\gamma(x, \theta)} f \psi_{\left(x_{0}, \theta_{0}\right)} d \sigma=0 \\
& \text { for }(x, \theta) \in \Omega\left(\mathcal{J}_{r\left(x_{0}, \theta_{0}\right), \varepsilon_{0}}\right),
\end{aligned}
$$


where $\Omega(\cdot)$ is defined in $(2.5), d=2, \mathcal{J}_{r, \varepsilon}$ is defined in (2.9). Formula (3.48) follows from (7.13).

Lemma 2 is proved.

\section{Proof of Lemma 3}

Proof of (6.1)-(6.3) Using (2.2), (3.13), (3.14), (3.45), (6.3) we obtain

$$
\begin{aligned}
& G(x, \theta)=\widetilde{G}(r(x, \theta))=\int_{\gamma(x, \theta)} f(x) d x, \\
& H_{k}(x, \theta)=\widetilde{H}_{k}(r(x, \theta))=\int_{\gamma(x, \theta)} f_{k}^{2}(x) d x, \\
& \tilde{f}_{k}(|x|)=\tilde{f}_{k}\left(\left(|x \theta|^{2}+|x-(x \theta) \theta|^{2}\right)^{1 / 2}\right), \quad(x, \theta) \in \Omega_{0}(1 / 2),
\end{aligned}
$$

where $\Omega_{0}(\cdot)$ is defined in $(2.3), d=2, \gamma(x, \theta)$ is defined as in (2.13).

Formulas (3.44), (3.45), (8.1)-(8.3) imply (6.1)-(6.3).

Proof of (6.4) Let

$$
\Lambda_{k}=\left(1-2^{-k+3}, 1-2^{-k+1}\right), \quad k \in \mathbb{N}, k \geq 4 .
$$

From (3.40) it follows that, for $k \geq 4$ :

$$
\begin{aligned}
& \operatorname{supp} \psi_{k-1} \subset\left(1-2^{-k+2}, 1-2^{-k}\right), \\
& \operatorname{supp} \psi_{k-2} \subset\left(1-2^{-k+3}, 1-2^{-k+1}\right)=\Lambda_{k}, \\
& \operatorname{supp} \psi_{k-3} \subset\left(1-2^{-k+4}, 1-2^{-k+2}\right) .
\end{aligned}
$$

Due to $(6.2),(6.3),(8.5)-(8.7)$, we have the following formula for $U_{0}$ :

$$
\begin{aligned}
U_{0}(r, s)=1 & -\widetilde{G}(r)\left((k-1) ! \tilde{f}_{k-1}\left(\left(s^{2}+r^{2}\right)^{1 / 2}\right) \frac{\psi_{k-3}(r)}{\widetilde{H}_{k-1}(r)}\right. \\
& +k ! \tilde{f}_{k}\left(\left(s^{2}+r^{2}\right)^{1 / 2}\right) \frac{\psi_{k-2}(r)}{\widetilde{H}_{k}(r)} \\
& \left.+(k+1) ! \tilde{f}_{k+1}\left(\left(s^{2}+r^{2}\right)^{1 / 2}\right) \frac{\psi_{k-1}(r)}{\widetilde{H}_{k+1}(r)}\right)
\end{aligned}
$$

for $r \in \Lambda_{k}, s \in \mathbb{R}, k \geq 4$. 
From (6.3), (8.8) it follows that

$$
\begin{gathered}
\frac{\partial^{n} U_{0}}{\partial s^{n}}(r, s)=-\widetilde{G}(r)\left((k-1) ! \frac{\partial^{n} \tilde{f}_{k-1}\left(\left(s^{2}+r^{2}\right)^{1 / 2}\right)}{\partial s^{n}} \frac{\psi_{k-3}(r)}{\widetilde{H}_{k-1}(r)}\right. \\
\quad+k ! \frac{\partial^{n} \tilde{f}_{k}\left(\left(s^{2}+r^{2}\right)^{1 / 2}\right)}{\partial s^{n}} \frac{\psi_{k-2}(r)}{\widetilde{H}_{k}(r)} \\
\left.\quad+(k+1) ! \frac{\partial^{n} \tilde{f}_{k+1}\left(\left(s^{2}+r^{2}\right)^{1 / 2}\right)}{\partial s^{n}} \frac{\psi_{k-1}(r)}{\widetilde{H}_{k+1}(r)}\right), \\
\quad \int_{-\infty}^{+\infty} \frac{\partial^{n}}{\partial r^{n}} \tilde{f}\left(\left(s^{2}+r^{2}\right)^{1 / 2}\right) d s, \\
\quad r^{n}(r)=\int_{-\infty}^{+\infty} \frac{\partial^{n}}{\partial r^{n}} \tilde{f}_{m}^{2}\left(\left(s^{2}+r^{2}\right)^{1 / 2}\right) d s, \\
r r^{n}(r)=\Lambda_{k}, \quad s \in \mathbb{R}, m \geq 1, n \geq 0, k \geq 4,
\end{gathered}
$$

where $\widetilde{G}, \widetilde{H}_{m}$ are defined in (6.3).

Using Lemma 1 and formulas (3.13), (3.14), (3.40)-(3.47), (6.3) one can see that:

$$
\begin{aligned}
& \tilde{f}, \tilde{f}_{m-2}, \widetilde{G}, \widetilde{H}_{m} \text { belong to } C_{0}^{\infty}(\mathbb{R}), \\
& \frac{\psi_{m-2}}{\widetilde{H}_{m}} \text { belongs to } C_{0}^{\infty}((1 / 2,1)) \text { for any } m \geq 3 .
\end{aligned}
$$

From (8.9)-(8.11) it follows that $U_{0}(r, s)$ has continuous partial derivatives of all orders with respect to $r \in \Lambda_{k}, s \in \mathbb{R}$. It implies that $U_{0} \in C^{\infty}\left(\Lambda_{k} \times \mathbb{R}\right)$. From the fact that $\Lambda_{k}, k \geq 4$, is an open cover of $(1 / 2,1)$ and from definition (6.2) of $U_{0}$, it follows that $U_{0} \in C^{\infty}(\{(1 / 2,1) \cup(1,+\infty)\} \times \mathbb{R})$.

This completes the proof of $(6.4)$.

Proof of (6.6) From (3.14)-(3.17) it follows that

$$
\tilde{f}_{k}(|x|)=0 \quad \text { if }|x| \geq 1 \text { for } k \in \mathbb{N} \text {. }
$$

Formula $|x|^{2}=|x \theta|^{2}+|x-(x \theta) \theta|^{2}, \quad x \in \mathbb{R}^{2}, \quad \theta \in \mathbb{S}^{1}$, and formulas (6.2), (8.12) imply (6.6). 
Proofs of (6.7)-(6.8)

Lemma 4. There are positive constants $c, k_{1}$ depending on $\Phi$ of (3.15)-(3.17), such that

(i) for all $k \in \mathbb{N}$ the following estimates hold:

$$
\begin{aligned}
& \left|\tilde{f}_{k}\right| \leq 1 \\
& \left|\tilde{f}_{k}^{\prime}\right| \leq c 8^{k},
\end{aligned}
$$

where $\tilde{f}_{k}^{\prime}$ denotes the derivative of $\tilde{f}_{k}$ defined in (6.3).

(ii) for $k \geq k_{1}$ and $1 / 2<r \leq 1$ the following estimates hold:

$$
\begin{aligned}
& \left|\frac{\psi_{k-2}(r)}{\widetilde{H}_{k}(r)}\right| \leq c 2^{k}, \\
& \left|\frac{d}{d r}\left(\frac{\psi_{k-2}(r)}{\widetilde{H}_{k}(r)}\right)\right| \leq c 2^{5 k},
\end{aligned}
$$

where $\psi_{k}$ are defined in (3.40), $\widetilde{H}_{k}$ is defined in (6.3).

(iii) for $k \geq 3$ and $r \geq 1-2^{-k}$ the following estimates hold:

$$
\begin{aligned}
& |\widetilde{G}(r)| \leq c \frac{(2 \sqrt{2})^{-k}}{k !}, \\
& \left|\frac{d \widetilde{G}}{d r}(r)\right| \leq c \frac{8^{k}}{k !},
\end{aligned}
$$

where $\widetilde{G}$ is defined in (6.3).

Lemma 5. Let $U_{0}$ be defined by (6.2)-(6.3). Then the following estimates are valid:

$$
\left|\frac{\partial U_{0}}{\partial s}(r, s)\right| \leq \frac{C}{(1-r)^{3}}, \quad\left|\frac{\partial U_{0}}{\partial r}(r, s)\right| \leq \frac{C}{(1-r)^{5}} \quad \text { for } r \in(1 / 2,1), s \in \mathbb{R}
$$

where $C$ is a constant depending only on $\Phi$ of (3.15)-(3.17).

Lemmas 4, 5 are proved in Subsections 9.1, 9.2, respectively. 
Proof of (6.7) From (8.15), (8.17) it follows that

$$
\begin{aligned}
& |\widetilde{G}(r)| \leq c(2 \sqrt{2})^{-k+3} /(k-3) !, \\
& \left|\frac{\psi_{k-2}(r)}{\widetilde{H}_{k}(r)}\right| \leq c 2^{k}
\end{aligned}
$$

for $r \in \Lambda_{k}, k \geq \max \left(4, k_{1}\right)$,

where $\Lambda_{k}$ is defined in (8.4).

Properties (8.5)-(8.7) and estimate (8.15) imply that

$$
\begin{aligned}
& \left\{\begin{array}{l}
\psi_{k-1}(r)=0, \\
\left|\frac{\psi_{k-3}(r)}{\widetilde{H}_{k-1}(r)}\right| \leq c 2^{k-1} \quad \text { if } r \in\left(1-2^{-k+3}, 1-2^{-k+2}\right),
\end{array}\right. \\
& \left\{\begin{array}{l}
\psi_{k-2}(r)=0, \\
\left|\frac{\psi_{k-1}(r)}{\widetilde{H}_{k+1}(r)}\right| \leq c 2^{k+1} \quad \text { if } r \in\left(1-2^{-k+2}, 1-2^{-k+1}\right),
\end{array}\right. \\
& \left\{\begin{array}{l}
\psi_{k-1}(r)=0, \\
\psi_{k-3}(r)=0
\end{array} \quad \text { if } r=1-2^{-k+2},\right.
\end{aligned}
$$

for $k \geq \max \left(4, k_{1}\right)$.

Note that the assumption that $r \in \Lambda_{k}$ is splitted into the assumptions on $r$ of (8.22), (8.23), (8.24).

Using formulas (8.8), (8.20)-(8.24), we obtain the following estimates:

$$
\begin{aligned}
\left|1-U_{0}(r, s)\right| & =|\widetilde{G}(r)|(k-1) ! \tilde{f}_{k-1}\left(\left(s^{2}+r^{2}\right)^{1 / 2}\right) \frac{\psi_{k-3}(r)}{\widetilde{H}_{k-1}(r)}+k ! \tilde{f}_{k}\left(\left(s^{2}+r^{2}\right)^{1 / 2}\right) \frac{\psi_{k-2}(r)}{\widetilde{H}_{k}(r)} \mid \\
& \leq c(2 \sqrt{2})^{-k+3}\left(c(k-2)(k-1) 2^{k-1}+c(k-2)(k-1) k 2^{k}\right)
\end{aligned}
$$

$\left|1-U_{0}(r, s)\right|=|\widetilde{G}(r)|\left|k ! \tilde{f}_{k}\left(\left(s^{2}+r^{2}\right)^{1 / 2}\right) \frac{\psi_{k-2}(r)}{\widetilde{H}_{k}(r)}+(k+1) ! \tilde{f}_{k+1}\left(\left(p^{2}+r^{2}\right)^{1 / 2}\right) \frac{\psi_{k-1}(r)}{\widetilde{H}_{k+1}(r)}\right|$

$$
\leq c(2 \sqrt{2})^{-k+3}\left(c 2^{k}(k-2)(k-1) k+c 2^{k+1}(k-2)(k-1) k(k+1)\right)
$$

$$
\leq 2^{10} \sqrt{2} c^{2} 2^{-k / 2} k^{4} \quad \text { if } r \in\left(1-2^{-k+2}, 1-2^{-k+1}\right),
$$


$\left|1-U_{0}(r, s)\right|=|\widetilde{G}(r)|\left|k ! \tilde{f}_{k}\left(\left(s^{2}+r^{2}\right)^{1 / 2}\right) \frac{\psi_{k-2}(r)}{\widetilde{H}_{k}(r)}\right|$

$$
\leq 2^{4} \sqrt{2} c^{2} 2^{-k / 2} k^{3} \quad \text { if } r=1-2^{-k+2},
$$

for $s \in \mathbb{R}, k \geq \max \left(4, k_{1}\right)$. Estimates (8.25)-(8.27) imply that

$$
\left|1-U_{0}(r, s)\right| \leq C 2^{-k / 2} k^{4}, \quad r \in \Lambda_{k}, s \in \mathbb{R}, k \geq \max \left(4, k_{1}\right),
$$

where $C$ is a positive constant depending on $c$ of Lemma 4 .

In addition, for $r \in \Lambda_{k}$ we have that $2^{-k+1}<(1-r)<2^{-k+3}$, which together with (8.28) imply (6.7).

This completes the proof of (6.7).

Proof of (6.8) We consider the following cases of $s, s^{\prime}, r, r^{\prime}$ in (6.8):

1. Let

$$
s, s^{\prime} \in \mathbb{R} \quad \text { and } \quad r, r^{\prime} \geq 1
$$

Due to (6.2) we have that

$$
U_{0}(r, s)=1, \quad U_{0}\left(r^{\prime}, s^{\prime}\right)=1 .
$$

Identities in (8.30) and assumption (8.29) imply (6.8) for this case.

2. Let

$$
s, s^{\prime} \in \mathbb{R}, \quad 1 / 2<r<1 \text { and } r^{\prime} \geq 1 .
$$

Then, due to (6.2), (6.7) we have that

$$
\begin{aligned}
& \left|1-U_{0}(r, s)\right| \leq C(1-r)^{1 / 3}, \\
& U_{0}\left(r^{\prime}, s^{\prime}\right)=1,
\end{aligned}
$$

where $s, s^{\prime}, r, r^{\prime}$ satisfy assumption (8.31), $C$ is a constant depending only on $\Phi$. In particular, inequality (8.32) follows from (6.7) due to the following simple property of the logarithm:

$$
\log _{2}^{a}\left(\frac{1}{1-r}\right) \leq C(a, \varepsilon)(1-r)^{-\varepsilon} \quad \text { for any } \varepsilon>0, r \in[0,1), a>0,
$$

where $C(a, \varepsilon)$ is some positive constant depending only on $a$ and $\varepsilon$.

Due to (8.31), (8.32), (8.33) we have that

$$
\begin{aligned}
\left|U_{0}\left(r^{\prime}, s^{\prime}\right)-U_{0}(r, s)\right| & =\left|1-U_{0}(r, s)\right| \leq C(1-r)^{1 / 3} \\
& \leq C\left|r-r^{\prime}\right|^{1 / 3} \leq C\left(\left|r-r^{\prime}\right|^{1 / 3}+\left|s-s^{\prime}\right|^{1 / 3}\right),
\end{aligned}
$$

where $C$ is a constant depending only on $\Phi$. 
Estimate (8.35) and assumptions (8.31) imply (6.8) for this case. Note that the case when $s, s^{\prime} \in \mathbb{R}, 1 / 2<r^{\prime}<1$ and $r \geq 1$ is completely similar to (8.31).

3. Let

$$
s, s^{\prime} \in \mathbb{R} \quad \text { and } \quad r, r^{\prime} \in(1 / 2,1) .
$$

In addition, without loss of generality we assume that $r>r^{\prime}$.

Next, using (6.4) one can see that

$$
\begin{aligned}
\left|U_{0}(r, s)-U_{0}\left(r^{\prime}, s^{\prime}\right)\right| & =\left|U_{0}(r, s)-U_{0}\left(r, s^{\prime}\right)+U_{0}\left(r, s^{\prime}\right)-U_{0}\left(r^{\prime}, s^{\prime}\right)\right| \\
& \leq\left|U_{0}(r, s)-U_{0}\left(r, s^{\prime}\right)\right|+\left|U_{0}\left(r, s^{\prime}\right)-U_{0}\left(r^{\prime}, s^{\prime}\right)\right| \\
& \leq\left|\frac{\partial U_{0}}{\partial s}(r, \hat{s})\right|\left|s-s^{\prime}\right|+\left|\frac{\partial U_{0}}{\partial r}\left(\hat{r}, s^{\prime}\right)\right|\left|r-r^{\prime}\right|,
\end{aligned}
$$

for $s, s^{\prime} \in \mathbb{R}, r, r^{\prime}>1 / 2$, and for appropriate $\hat{s}, \hat{r}$.

Note that $\hat{s}, \hat{r}$ belong to open intervals $\left(s, s^{\prime}\right),\left(r^{\prime}, r\right)$, respectively.

Using (6.7), (8.19), (8.32), (8.37) and the property that $1 / 2<r^{\prime}<\hat{r}<r<1$ we obtain

$$
\begin{aligned}
& \left|U_{0}(r, s)-U_{0}\left(r^{\prime}, s^{\prime}\right)\right| \leq C\left((1-r)^{1 / 3}+\left(1-r^{\prime}\right)^{1 / 3}\right), \\
& \left|U_{0}(r, s)-U_{0}\left(r^{\prime}, s^{\prime}\right)\right| \leq \frac{C}{(1-r)^{5}}\left(\left|s-s^{\prime}\right|+\left|r-r^{\prime}\right|\right),
\end{aligned}
$$

where $C$ is a constant depending only on $\Phi$.

We have that

$$
\begin{aligned}
(1-r)^{1 / 3}+\left(1-r^{\prime}\right)^{1 / 3} & =(1-r)^{1 / 3}+\left((1-r)+\left(r-r^{\prime}\right)\right)^{1 / 3} \\
& \leq 2(1-r)^{1 / 3}+\left|r-r^{\prime}\right|^{1 / 3} \\
& \leq \begin{cases}3\left|r-r^{\prime}\right|^{1 / 3} & \text { if } 1-r \leq\left|r-r^{\prime}\right| \\
3(1-r)^{1 / 3} & \text { if } 1-r>\left|r-r^{\prime}\right|\end{cases}
\end{aligned}
$$

where $r, r^{\prime}$ satisfy (8.36). Note that in (8.40) we used the following inequality:

$$
(a+b)^{1 / m} \leq a^{1 / m}+b^{1 / m} \quad \text { for } a \geq 0, b \geq 0, m \in \mathbb{N} .
$$

In particular, using (8.38), (8.40) we have that

$$
\left|U_{0}(r, s)-U_{0}\left(r^{\prime}, s^{\prime}\right)\right|^{15} \leq 3^{15} C^{15}(1-r)^{5} \text { if } 1-r>\left|r-r^{\prime}\right|,
$$

where $s, s^{\prime}, r, r^{\prime}$ satisfy assumption (8.36), $C$ is a constant of (8.38), (8.39).

Multiplying the left and the right hand-sides of (8.39), (8.42) we obtain

$$
\left|U_{0}(r, s)-U_{0}\left(r^{\prime}, s^{\prime}\right)\right|^{16} \leq 3^{15} C^{16}\left(\left|s-s^{\prime}\right|+\left|r-r^{\prime}\right|\right), \quad \text { if } 1-r>\left|r-r^{\prime}\right| .
$$


Using (8.38), (8.40) we obtain

$$
\left|U_{0}(r, s)-U_{0}\left(r^{\prime}, s^{\prime}\right)\right| \leq 3 C\left|r-r^{\prime}\right|^{1 / 3}, \quad \text { if } 1-r \leq\left|r-r^{\prime}\right|,
$$

where $C$ is a constant of (8.38), (8.39) depending only on $\Phi$. Using (8.43) and (8.41) for $m=16, a=\left|s-s^{\prime}\right|, b=\left|r-r^{\prime}\right|$, we have that

$$
\left|U_{0}(r, s)-U_{0}\left(r^{\prime}, s^{\prime}\right)\right| \leq 3 C\left(\left|s-s^{\prime}\right|^{1 / 16}+\left|r-r^{\prime}\right|^{1 / 16}\right), \quad \text { if } 1-r>\left|r-r^{\prime}\right|,
$$

where $s, s^{\prime}, r, r^{\prime}$ satisfy assumption (8.36), $C$ is a constant of (8.38), (8.39) which depends only on $\Phi$.

Formulas (8.44), (8.45) imply (6.8) for this case.

Note that assumptions $(8.29),(8.31),(8.36)$ for cases $1,2,3$, respectively, cover all possible choices of $s, s^{\prime}, r, r^{\prime}$ in (6.8).

This completes the proof of (6.8).

This completes the proof of Lemma 3.

\section{Proofs of Lemmas 4,5}

\subsection{Proof of Lemma 4}

Proof of (8.13), (8.14) Estimates (8.13), (8.14) follow directly from (3.14)(3.17).

Proof of (8.17) We will use the following parametrization of the points $y$ on $\gamma(x, \theta) \in T \mathbb{S}^{1}, \quad(x, \theta) \in \Omega, r(x, \theta) \neq 0$ (see notations $(2.1),(2.2),(2.13)$ for $\left.d=2\right)$ :

$$
y(\beta)=x-(x \theta) \theta+\tan (\beta) r(x, \theta) \theta, \quad \beta \in(-\pi / 2, \pi / 2),
$$

where $\beta$ is the parameter.

We have that:

$$
d \sigma(\beta)=r d(\tan (\beta))=\frac{r d \beta}{\cos ^{2} \beta}, \quad r=r(x, \theta),
$$

where $\sigma$ is the standard Lebesgue measure on $\gamma(x, \theta)$.

From definitions (3.13), (6.3) it follows that

$$
\begin{aligned}
& \widetilde{G}(r)=\sum_{k=1}^{\infty} \frac{\widetilde{G}_{k}(r)}{k !}, \\
& \widetilde{G}_{k}(r)=\int_{\gamma_{r}} \tilde{f}_{k}(|y|) d y, \quad \gamma_{r} \in T(r), r>1 / 2,
\end{aligned}
$$

where $T(r)$ is defined by (2.8). 
Using (3.14), (9.1), (9.2), (9.4) we obtain the following formula for $\widetilde{G}_{k}$ :

$$
\begin{aligned}
\widetilde{G}_{k}(r)= & r \int_{-\pi / 2}^{\pi / 2} \Phi\left(2^{k}\left(1-\frac{r}{\cos \beta}\right)\right) \cos \left(8^{k} \frac{r^{2}}{\cos ^{2} \beta}\right) \frac{d \beta}{\cos ^{2} \beta} \\
= & \{u=\tan (\beta)\}=2 r \int_{0}^{+\infty} \Phi\left(2^{k}\left(1-r \sqrt{u^{2}+1}\right)\right) \cos \left(8^{k} r^{2}\left(u^{2}+1\right)\right) d u \\
= & \left\{t=u^{2}\right\}=r \int_{0}^{+\infty} \Phi\left(2^{k}(1-r \sqrt{t+1})\right) \cos \left(8^{k} r^{2}(t+1)\right) \frac{d t}{\sqrt{t}} \\
= & r \cos \left(8^{k} r^{2}\right) \int_{0}^{+\infty} \Phi\left(2^{k}(1-r \sqrt{t+1})\right) \frac{\cos \left(8^{k} r^{2} t\right)}{\sqrt{t}} d t \\
& -r \sin \left(8^{k} r^{2}\right) \int_{0}^{+\infty} \Phi\left(2^{k}(1-r \sqrt{t+1})\right) \frac{\sin \left(8^{k} r^{2} t\right)}{\sqrt{t}} d t \\
= & 8^{-k / 2} r^{-1} \cos \left(8^{k} r^{2}\right) \int_{0}^{+\infty} \Phi_{k}(t, r) \frac{\cos (t)}{\sqrt{t}} d t \\
& \left.-8^{-k / 2} r^{-1} \sin ^{+\infty} 8^{k} r^{2}\right) \int_{0}^{+\infty}(t, r) \frac{\sin (t)}{\sqrt{t}} d t, \quad r>1 / 2,
\end{aligned}
$$

where

$$
\Phi_{k}(t, r)=\Phi\left(2^{k}\left(1-r \sqrt{8^{-k} r^{-2} t+1}\right)\right), \quad t \geq 0, r>1 / 2, k \in \mathbb{N} .
$$

For integrals arising in (9.5) the following estimates hold:

$$
\begin{aligned}
& \left|\int_{0}^{+\infty} \Phi_{k}(t, r) \frac{\sin (t)}{\sqrt{t}} d t\right| \leq C_{1}<+\infty, \\
& \left|\int_{0}^{+\infty} \Phi_{k}(t, r) \frac{\cos (t)}{\sqrt{t}} d t\right| \leq C_{2}<+\infty, \\
& \quad \text { for } 1 / 2<r<1, k \geq 1 .
\end{aligned}
$$

where $\Phi_{k}$ is defined in (9.6), $C_{1}, C_{2}$ are some positive constants depending only on $\Phi$ and not depending on $k$ and $r$.

Estimates (9.7), (9.8) are proved in Subsection 9.3.

From (9.5)-(9.8) it follows that

$$
\left|\widetilde{G}_{k}(r)\right| \leq 2 \cdot 8^{-k / 2}\left(C_{1}+C_{2}\right) \quad \text { for } r>1 / 2, k \in \mathbb{N} .
$$


Note that for $y \in \gamma_{r}$, the following inequality holds:

$$
\begin{aligned}
& 2^{k}(1-|y|) \leq 2^{k}(1-r) \leq 2^{k-m} \leq 1 / 2<4 / 5 \\
& \quad \text { for } 1-2^{-m} \leq r<1, k<m, m \geq 3,
\end{aligned}
$$

where $\gamma_{r}$ is a ray in $T(r)$ (see notations of $(2.8), d=2$ ).

Formulas (3.14), (3.15), (6.3), (9.10) imply that

$$
\gamma_{r} \cap \operatorname{supp} f_{k}=\varnothing \quad \text { if } r \geq 1-2^{-m}, k<m,
$$

In turn, (9.4), (9.11) imply that

$$
\widetilde{G}_{k}(r)=0 \quad \text { for } r \geq 1-2^{-m}, k<m, m \geq 3 .
$$

Due to (9.3), (9.4), (9.9), (9.12) we have that:

$$
\begin{aligned}
& |\widetilde{G}(r)| \leq \sum_{k=m}^{\infty}\left|\widetilde{G}_{k}(r)\right| / k ! \\
& \quad \leq 2\left(C_{1}+C_{2}\right) \frac{(2 \sqrt{2})^{-m}}{m !} \sum_{k=0}^{\infty}(2 \sqrt{2})^{-k}=c_{1} \frac{(2 \sqrt{2})^{-m}}{m !}, \\
& c_{1}=\left(C_{1}+C_{2}\right) \frac{4 \sqrt{2}}{2 \sqrt{2}-1}, \\
& \quad \text { for } r \geq 1-2^{-m}, m \geq 3 .
\end{aligned}
$$

This completes the proof of estimate (8.17).

Proof of (8.18) Using (9.3), (9.4) we have that:

$$
\left|\frac{d \widetilde{G}}{d r}(r)\right| \leq \sum_{k=1}^{\infty} \frac{1}{k !}\left|\frac{d \widetilde{G}_{k}(r)}{d r}\right| .
$$

Formulas (3.14), (8.10) for $n=1,(8.14)$, (9.4) imply that

$$
\begin{aligned}
\left|\frac{d \widetilde{G}_{k}}{d r}(r)\right| & =\left|\int_{-\infty}^{+\infty} \frac{r \tilde{f}_{k}^{\prime}\left(\left(s^{2}+r^{2}\right)^{1 / 2}\right)}{\sqrt{r^{2}+s^{2}}} d s\right| \\
& \leq \int_{-\infty}^{+\infty}\left|\tilde{f}_{k}^{\prime}\left(\left(s^{2}+r^{2}\right)^{1 / 2}\right)\right| d s=\int_{\gamma_{r}}\left|\tilde{f}_{k}^{\prime}(|y|)\right| d y \\
& \leq c 8^{k} \int_{\gamma_{r} \cap B(0,1)} d y \leq 2 c 8^{k}
\end{aligned}
$$

where $B(0,1)$ is defined in $(2.16), d=2$. 
At the same time, formula (9.12) implies that

$$
\frac{d \widetilde{G}_{k}(r)}{d r}=0 \quad \text { for } r \geq 1-2^{-m}, k<m, m \geq 3 .
$$

Formulas (9.14), (9.15), (9.16) imply the following sequence of inequalities:

$$
\left|\frac{d \widetilde{G}(r)}{d r}\right| \leq \sum_{k=m}^{\infty} \frac{1}{k !}\left|\frac{d \widetilde{G}_{k}(r)}{d r}\right| \leq c \frac{8^{m}}{m !} \sum_{k=0}^{\infty} \frac{m ! 8^{k}}{(k+m) !}, \quad r \geq 1-2^{-m}, m \geq 3 .
$$

The series in the right hand-side in (9.17) admits the following estimate:

$$
\sum_{k=0}^{\infty} \frac{m ! 8^{k}}{(k+m) !} \leq \sum_{k=0}^{\infty} \frac{8^{k}}{k !}=e^{8} \quad \text { and the estimate does not depend on } m .
$$

Formulas (9.17), (9.18) imply (8.18).

Proof of (8.15) For each $\psi_{k}$ from (3.40) we have that

$$
\left|\psi_{k}\right| \leq 1
$$

Therefore, it is sufficient to show that

$$
\widetilde{H}_{k} \geq C 2^{-k} \text { for } k \geq k_{1}, C=c^{-1} .
$$

Proceeding from (6.3) and in a similar way with (9.5) we obtain the formulas

$$
\begin{aligned}
\widetilde{H}_{k}(r) & =r \int_{0}^{+\infty} \frac{\Phi^{2}\left(2^{k}(1-r \sqrt{t+1})\right)}{\sqrt{t}} \cos ^{2}\left(8^{k} r^{2}(t+1)\right) d t \\
& =\widetilde{H}_{k, 1}(r)+\widetilde{H}_{k, 2}(r), \quad r>1 / 2, \\
\widetilde{H}_{k, 1}(r) & =\frac{r}{2} \int_{0}^{+\infty} \frac{\Phi^{2}\left(2^{k}(1-r \sqrt{t+1})\right)}{\sqrt{t}} d t \\
\widetilde{H}_{k, 2}(r) & =\frac{r}{2} \int_{0}^{+\infty} \frac{\Phi^{2}\left(2^{k}(1-r \sqrt{t+1})\right)}{\sqrt{t}} \cos \left(2 \cdot 8^{k} r^{2}(t+1)\right) d t .
\end{aligned}
$$

In addition, we have that:

$$
\operatorname{supp}_{t} \Phi^{2}\left(2^{k}(1-r \sqrt{t+1})\right) \subset[0,3] \text { for } 1 / 2<r \leq 1-2^{-k+1}, k \geq 3,
$$


where $\operatorname{supp}_{t}$ denotes the support of the function in variable $t$. Property (9.24) is proved below in this paragraph (see formulas (9.26)-(9.29)).

Note that

$$
2^{k}(1-r) \geq 2^{k} \cdot 2^{-k+1} \geq 2>6 / 5 \text { for } 1 / 2<r \leq 1-2^{-k+1}, k \geq 3 .
$$

From (3.15), (3.16) and from (9.25) we have that:

$$
\operatorname{supp}_{t} \Phi^{2}\left(2^{k}(1-r \sqrt{t+1})\right) \subset[0,+\infty) \quad \text { for } 1 / 2<r \leq 1-2^{-k+1}, k \geq 3 .
$$

We have that

$$
\begin{aligned}
& \exists t_{1}^{(k)}=t_{1}^{(k)}(r) \geq 0, \quad t_{2}^{(k)}=t_{2}^{(k)}(r) \geq 0, t_{2}^{(k)}>t_{1}^{(k)}, \text { such that } \\
& \left\{\begin{array}{l}
2^{k}\left(1-r \sqrt{t_{1}^{(k)}+1}\right)=11 / 10, \\
2^{k}\left(1-r \sqrt{t_{2}^{(k)}+1}\right)=9 / 10,
\end{array}\right. \\
& \quad\left|t_{2}^{(k)}-t_{1}^{(k)}\right| \geq\left(\sqrt{t_{2}^{(k)}+1}-\sqrt{t_{1}^{(k)}+1}\right)=\frac{2^{-k}}{5} r^{-1} \geq \frac{2^{-k}}{5}, \\
& \quad \text { for } 1 / 2<r \leq 1-2^{-k+1}, k \geq 3 .
\end{aligned}
$$

In addition, from (9.27) it follows that

$$
\begin{aligned}
& t_{1}^{(k)}=\frac{\left(1-2^{-k} \frac{11}{10}\right)^{2}}{r^{2}}-1 \leq 4\left(1-2^{-k} \frac{11}{10}\right)^{2}-1 \leq 3, \\
& t_{2}^{(k)}=\frac{\left(1-2^{-k} \frac{9}{10}\right)^{2}}{r^{2}}-1 \leq 4\left(1-2^{-k} \frac{11}{10}\right)^{2}-1 \leq 3, \\
& \quad \text { for } 1 / 2<r \leq 1-2^{-k+1}, k \geq 3 .
\end{aligned}
$$

Using (3.15)-(3.17), (9.22), (9.24), (9.27)-(9.29) we have that

$$
\widetilde{H}_{k, 1}(r) \geq \frac{r}{2} \int_{t_{1}^{(k)}}^{t_{2}^{(k)}} \frac{d t}{\sqrt{t}} \geq \frac{r}{2} \int_{1+t_{1}^{(k)}}^{1+t_{2}^{(k)}} \frac{d t}{\sqrt{t}}
$$

$$
\geq r\left(\sqrt{t_{2}^{(k)}+1}-\sqrt{t_{1}^{(k)}+1}\right) \geq \frac{2^{-k}}{10} \text { for } 1 / 2<r \leq 1-2^{-k+1}, k \geq 3 .
$$

On the other hand, proceeding from using (9.23) and, in a similar way with (9.5)(9.9), we have

$$
\left|\widetilde{H}_{k, 2}(r)\right|=\frac{r}{2}\left|\int_{0}^{+\infty} \frac{\Phi^{2}\left(2^{k}(1-r \sqrt{t+1})\right)}{\sqrt{t}} \cos \left(2 \cdot 8^{k} r^{2}(t+1)\right) d t\right|
$$




$$
\begin{aligned}
& \leq \frac{r}{2}\left|\cos \left(2 \cdot 8^{k} r^{2}\right)\right|\left|\int_{0}^{+\infty} \Phi^{2}\left(2^{k}(1-r \sqrt{t+1})\right) \frac{\cos \left(2 \cdot 8^{k} r^{2} t\right)}{\sqrt{t}} d t\right| \\
& \quad+\frac{r}{2}\left|\sin \left(2 \cdot 8^{k} r^{2}\right)\right|\left|\int_{0}^{+\infty} \Phi^{2}\left(2^{k}(1-r \sqrt{t+1})\right) \frac{\sin \left(2 \cdot 8^{k} r^{2} t\right)}{\sqrt{t}} d t\right| \\
& \leq 8^{-k / 2} \frac{r^{-1}}{2}\left|\int_{0}^{+\infty} \Phi_{k}^{2}(t, r) \frac{\cos (2 t)}{\sqrt{t}} d t\right|+8^{-k / 2} \frac{r^{-1}}{2}\left|\int_{0}^{+\infty} \Phi_{k}^{2}(t, r) \frac{\sin (2 t)}{\sqrt{t}} d t\right| \\
& \leq 8^{-k / 2} C, \quad \text { for } 1 / 2<r<1-2^{-k+1}, k \geq 3,
\end{aligned}
$$

where $\Phi_{k}(t, r)$ is defined in (9.6), $C$ is some constant depending only on $\Phi$ and not depending on $k, r$. In $(9.31)$ we have also used that $\Phi^{2}(t)$ satisfies assumptions (3.15)-(3.17).

Note also that $\Phi^{2}(t)$ satisfies assumptions (3.15)-(3.17) for $\Phi(t)$.

Using (9.21)-(9.23), (9.30), (9.31) we obtain

$$
\begin{aligned}
\left|\widetilde{H}_{k}(r)\right| & \geq\left|\widetilde{H}_{k, 1}(r)\right|-\left|\widetilde{H}_{k, 2}(r)\right| \\
& \geq \frac{2^{-k}}{10}-C^{\prime} \cdot 8^{-k / 2} \\
& \geq 2^{-k}\left(\frac{1}{10}-\frac{C^{\prime}}{(\sqrt{2})^{k}}\right) \\
& \geq C \cdot 2^{-k} \text { for } 1 / 2<r<1-2^{-k+1}, k \geq k_{1} \geq 3, \\
C & =\frac{1}{10}-C^{\prime}(\sqrt{2})^{-k_{1}},
\end{aligned}
$$

where $C^{\prime}$ depends only on $\Phi, k_{1}$ is arbitrary constant such that $k_{1} \geq 3$ and $C$ is positive.

Formulas (8.15) follows from (3.40), (9.32).

This completes the proof $(8.15)$.

Proof of (8.16) The following formula holds:

$$
\frac{d}{d r}\left(\frac{\psi_{k-2}(r)}{\widetilde{H}_{k}(r)}\right)=-\frac{\widetilde{H}_{k}^{\prime}(r) \psi_{k-2}(r)-\widetilde{H}_{k}(r) \psi_{k-2}^{\prime}(r)}{\widetilde{H}_{k}^{2}(r)}, 1 / 2<r<1,
$$

where $\widetilde{H}_{k}^{\prime}, \psi_{k-2}^{\prime}$ denote the derivatives of $\widetilde{H}_{k}, \psi_{k}$, defined in (6.3), (3.40), respectively. 
Using (3.14), (6.3), (8.10), $n=1,(8.13),(8.14)$ we have that

$$
\begin{aligned}
\left|\tilde{H}_{k}^{\prime}(r)\right| & =2\left|\int_{-\infty}^{+\infty} \frac{r}{\sqrt{r^{2}+s^{2}}} \tilde{f}_{k}\left(\sqrt{r^{2}+s^{2}}\right) \tilde{f}_{k}^{\prime}\left(\sqrt{r^{2}+s^{2}}\right) d s\right| \\
& \leq 2 \int_{-\infty}^{+\infty}\left|\tilde{f}_{k}\left(\sqrt{r^{2}+s^{2}}\right) \tilde{f}_{k}^{\prime}\left(\sqrt{r^{2}+s^{2}}\right)\right| d s=2 \int_{\gamma_{r}}\left|\tilde{f}_{k}(|y|) \tilde{f}_{k}^{\prime}(|y|)\right| d y \\
& \leq 2 c 8^{k} \int_{\gamma_{r} \cap B(0,1)} d y \leq 4 c 8^{k}, \quad \gamma_{r} \in T(r), k \geq 3, r>1 / 2,
\end{aligned}
$$

where we use notations $(2.8),(2.16), d=2$.

Using (3.40), (3.41), (8.15), (9.32)-(9.34) we have that

$$
\begin{aligned}
& \left|\frac{d}{d r}\left(\frac{\psi_{k-2}(r)}{\widetilde{H}_{k}(r)}\right)\right| \leq C 2^{2 k}\left(\left|\widetilde{H}_{k}^{\prime}(r)\right|+\left|\widetilde{H}_{k}(r)\right| \cdot\left|\psi_{k}^{\prime}(r)\right|\right) \leq C^{\prime} 2^{5 k}, \\
& \quad \text { for } 1 / 2<r<1-2^{-k+1}, k \geq k_{1} \geq 3,
\end{aligned}
$$

where $C^{\prime}$ is a constant not depending on $k$ and $r$ and depending only on $\Phi$.

This completes the proof of Lemma 4.

\subsection{Proof of Lemma 5}

It is sufficient to show that

$$
\begin{aligned}
& \left|\frac{\partial U_{0}(r, s)}{\partial s}\right| \leq \frac{C}{(1-r)^{3}}, \\
& \left|\frac{\partial U_{0}(r, s)}{\partial r}\right| \leq \frac{C}{(1-r)^{5}}, \\
& \quad \text { for } s \in \mathbb{R}, r \in \Lambda_{k}, k \geq \max \left(4, k_{1}\right),
\end{aligned}
$$

where $C$ is a positive constant depending only on $\Phi$ of (3.14), $\Lambda_{k}$ is defined in (8.4), $k_{1}$ is a constant arising in Lemma 4 and depending only on $\Phi$.

Indeed, estimates (8.19) follow from (6.4), (9.36), (9.37) and the fact that $\Lambda_{k}, k \geq 4$, is an open cover of $(1 / 2,1)$.

In turn, estimates $(9.36),(9.37)$ follow from the estimates

$$
\left|\frac{\partial U_{0}(r, s)}{\partial s}\right| \leq C \cdot 8^{k},
$$




$$
\begin{gathered}
\left|\frac{\partial U_{0}(r, s)}{\partial r}\right| \leq C \cdot(32)^{k}, \\
\text { for } s \in \mathbb{R}, r \in \Lambda_{k},
\end{gathered}
$$

and from the fact that $2^{-k+1}<1-r<2^{-k+3}, k \geq \max \left(4, k_{1}\right)$, for $r \in \Lambda_{k}$, where $C$ is a positive constant depending only on $\Phi$.

Estimate (9.38) follows from formula (8.9) for $n=1$ and estimates (8.14), (8.15), (8.20)-(8.24).

Estimate (9.39) follows from (8.8), (8.13)-(8.16), (8.20)-(8.24) and from the estimates:

$$
\begin{aligned}
& \left|\frac{d}{d r}\left(\frac{\psi_{k-i}(r)}{\widetilde{H}_{k-i+2}(r)}\right)\right| \leq c 2^{5(k+1)}, \\
& \left|\frac{d \widetilde{G}(r)}{d r}\right| \leq c \frac{8^{-k+3}}{(k-3) !}, \\
& \quad \text { for } r \in \Lambda_{k}, \quad i \in\{1,2,3\},
\end{aligned}
$$

where $c$ is a constant arising in Lemma 4 .

Estimate (9.40) follows from (8.16) (used with $k-1, k, k+1$ in place of $k$ ). Estimate (9.41) follows from (8.18) (used with $k-3$ in place of $k$ ).

This completes the proof of Lemma 5 .

\subsection{Proof of estimates $(9.7),(9.8)$}

We use the following Bonnet's integration formulas (see, e.g., [F59], Chapter 2):

$$
\begin{aligned}
& \int_{a}^{b} f_{1}(t) h(t) d t=f_{1}(a) \int_{a}^{\xi_{1}} h(t) d t \\
& \int_{a}^{b} f_{2}(t) h(t) d t=f_{2}(b) \int_{\xi_{2}}^{b} h(t) d t
\end{aligned}
$$

for some appropriate $\xi_{1}, \xi_{2} \in[a, b]$, where

$$
\begin{aligned}
& f_{1} \text { is monotonously decreasing on }[a, b], f_{1} \geq 0, \\
& f_{2} \text { is monotonously increasing on }[a, b], f_{2} \geq 0, \\
& h(t) \text { is integrable on }[a, b] .
\end{aligned}
$$


Let

$$
\begin{aligned}
& g_{1}(t)=\frac{\sin (t)}{\sqrt{t}}, \quad g_{2}(t)=\frac{\cos (t)}{\sqrt{t}}, t>0, \\
& G_{1}(s)=\int_{0}^{s} \frac{\sin (t)}{\sqrt{t}} d t, \quad G_{2}(s)=\int_{0}^{s} \frac{\cos (t)}{\sqrt{t}} d t, s \geq 0 .
\end{aligned}
$$

We recall that

$$
\lim _{s \rightarrow+\infty} G_{1}(s)=\lim _{s \rightarrow+\infty} G_{2}(s)=\sqrt{\frac{\pi}{2}} .
$$

From (9.45), (9.46), (9.47) it follows that

$$
G_{1}, G_{2} \text { are continuous and bounded on }[0,+\infty) \text {. }
$$

Due to $(3.15)-(3.18),(9.6)$ and monotonicity of the function $2^{k}\left(1-r \sqrt{8^{-k} r^{-2} t+1}\right)$ in $t$ on $[0,+\infty)$ it follows that

(9.49) $\Phi_{k}(t, r)$ is monotonously decreasing on $[0,+\infty)$, if $2^{k}(1-r) \leq 11 / 10$,

(9.50) $\Phi_{k}(t, r)$ is monotonously increasing on $\left[0, t_{0}\right]$ for some $t_{0}>0$ and is monotonously decreasing on $\left[t_{0},+\infty\right)$, if $2^{k}(1-r)>11 / 10$. for $r>1 / 2, k \in \mathbb{N}$,

Moreover, due to (3.15)-(3.17), (9.6), for $T_{k}=8^{k}, k \in \mathbb{N}$, we have that

$$
\begin{aligned}
& \Phi_{k}\left(T_{k}, r\right)=\Phi\left(2^{k}\left(1-r \sqrt{r^{-2}+1}\right)\right)=\Phi\left(2^{k}\left(1-\sqrt{1+r^{-2}}\right)\right)=0 \\
& \Phi_{k}(t, r)=0 \quad \text { for } t \geq T_{k} \\
& \left|\Phi_{k}(t, r)\right| \leq 1 \quad \text { for } t \geq 0 \\
& \quad r>1 / 2, \quad k \in \mathbb{N} .
\end{aligned}
$$

Using (9.6), (9.45)-(9.50), (9.52) and (9.42)-(9.44) we obtain

$$
\begin{aligned}
\int_{0}^{+\infty} \Phi_{k}(t, r) g_{i}(t) d t & =\int_{0}^{T_{k}} \Phi_{k}(t, r) g_{i}(t) d t=\Phi_{k}(0, r) \int_{0}^{\xi} g_{i}(t) d t \\
& =\Phi_{k}(0, r) G_{i}(\xi) \text { for appropriate } \xi \in\left[0, T_{k}\right],
\end{aligned}
$$

(9.54) $\quad$ if $2^{k}(1-r) \leq 11 / 10$

$$
\int_{0}^{\infty} \Phi_{k}(t, r) g_{i}(t) d t=\int_{0}^{T_{k}} \Phi_{k}(t, r) g_{i}(t) d t=\int_{0}^{t_{0}} \Phi_{k}(t, r) g_{i}(t) d t
$$




$$
\begin{gathered}
+\int_{t_{0}}^{T_{k}} \Phi_{k}(t, r) g_{i}(t) d t \\
=\Phi_{k}\left(t_{0}, r\right) \int_{\xi^{\prime}}^{t_{0}} g_{i}(t) d t+\Phi_{k}\left(t_{0}, r\right) \int_{t_{0}}^{\xi^{\prime \prime}} g_{i}(t) d t \\
=\Phi_{k}\left(t_{0}, r\right)\left(G_{i}\left(\xi^{\prime \prime}\right)-G_{i}\left(\xi^{\prime}\right)\right) \\
\text { for appropriate } \xi^{\prime} \in\left[0, t_{0}\right], \xi^{\prime \prime} \in\left[t_{0}, T_{k}\right], \text { if } 2^{k}(1-r)>11 / 10,
\end{gathered}
$$

where $i=\overline{1,2}$.

Estimates (9.7), (9.8) follow from (9.45), (9.46), (9.48), (9.53)-(9.55).

\section{References}

[ABK98] Arbuzov, E. V., Bukhgeim, A. L. and Kazantsev, S. G., Two-dimensional tomography problems and the theory of A-analytic functions, Siberian Adv. Math. 8 (1998), 1-20. MR1666500

[Bal09] BAL, G., Inverse transport theory and applications, Inverse Probl. 25, 053001 (2009), 48 pp. MR2501018

[BJ11] BAL, G. and Jollivet, A., Combined source and attenuation reconstructions in SPECT, in Tomography and Inverse Transport Theory, Contemp. Math. 559, pp. 13-27, 2011. MR2885192

[Be84] Beylkin, G., The inversion problem and applications of the generalized Radon transform, Comm. Pure Appl. Math. 37 (1984), 579-599. MR0752592

[BQ87] Boman, J. and Quinto, E. T., Support theorems for real-analytic Radon transforms, Duke Math. J. 55 (1987), 943-948. MR0916130

[BQ93] Boman, J. and Quinto, E. T., Support theorems for Radon transforms on real analytic line complexes in three-space, Trans. Amer. Math. Soc. 335 (1993), 877-890. MR1080733

[B93] Boman, J., An example of non-uniqueness for a generalized Radon transform, J. Anal. Math. 61 (1993), 395-401. MR1253450

[BS04] Boman, J. and Strömberg, J.-O., Novikov's inversion formula for the attenuated Radon transform-a new approach, J. Geom. Anal. 14 (2004), 185-198. MR2051682

[C64] CoRmack, A. M., Representation of a function by its line integrals, with some radiological applications I, II, J. Appl. Phys. 34 (1963), 2722-2727; 35 (1964) 2908-2912

[M92] Do CARmo, M. P., Riemannian Geometry, Birkhäuser, Basel, 1992. MR1138207

[F59] Fichtenholz, G. M., A course of differential and integral calculus, volume II, Fizmatgiz, Moscow, 1959. MR0524565

[Fi86] Finch, D., Uniqueness for the attenuated X-ray transform in the physical range, Inverse Probl. 2 (1986). MR0847534 
[GGG82] Gel'Fand, I. M., Gindikin, S. G. and Graev, M. I., Integral geometry in affine and projective spaces, J. Sov. Math. 18 (1982), 39-167.

[Gi10] Gindikin, S., A remark on the weighted Radon transform on the plane, Inverse Probl. Imaging 4 (2010), 649-653. MR2726420

[GN18] Goncharov, F. O. and Novikov, R. G., An example of non-uniqueness for Radon transforms with continuous positive rotation invariant weights, J. Geom. Anal. 28 (2018), 3807-3828. MR3881991

[H01] Helgason, S., Differential geometry and symmetric spaces 341, American Mathematical Soc., Providence, 2001. MR1834454

[I16] Ilmavirta, J., Coherent quantum tomography, SIAM J. Math. Anal. 48 (2016), 3039-3064. MR3544653

[J38] John, F., The ultrahyperbolic differential equation with 4 independent variables, Duke Math. J. 4 (1938), 300-322. MR1546052

[KLM95] Kuchment, P., Lancaster, K. and Mogilevskaya, L., On local tomography, Inverse Probl. 11 (1995), 571-589. MR1333096

[Kun92] Kunyansky, L., Generalized and attenuated Radon transforms: restorative approach to the numerical inversion, Inverse Probl. 8 (1992), 809819. MR1185600

[LB73] Lavrent'ev, M. M. and Bukhgeim, A. L., A class of operator equations of the first kind, Funct. Anal. Appl. 7 (1973), 290-298. MR0343106

[MQ85] Markoe, A. and Quinto, E. T., An elementary proof of local invertibility for generalized and attenuated Radon transforms, SIAM J. Math. Anal. 16 (1985), 1114-1119. MR0800800

[Na01] NatTerer, F., The Mathematics of Computerized Tomography, SIAM, Philadelphia, 2001. MR1847845

[Ng17] NGUYEN, L. V., On the strength of streak artifacts in filtered back-projection reconstructions for limited angle weighted X-ray transform, J. Fourier Anal. Appl. 23 (2017), 712-728. MR3649477

[N02a] Novikov, R. G., On determination of a gauge field on $\mathbb{R}^{d}$ from its non-abelian Radon transform along oriented straight lines, J. Inst. Math. Jussieu 1 (2002), 559-629. MR1954437

[N02b] Novikov, R. G., An inversion formula for the attenuated X-ray transformation, Ark. Mat. 40 (2002), 145-167. MR1948891

[N14] Novikov, R. G., Weighted Radon transforms and first order differential systems on the plane, Mosc. Math. J. 14 (2014), 807-823. MR3292050

[Pa96] Palamodov, V. P., An inversion method for an attenuated x-ray transform, Inverse Probl. 12 (1996), 717-729. MR1413429

[PG13] Puro, A. and Garin, A., Cormack-type inversion of attenuated Radon transform, Inverse Probl. 29, 065004 (2013). 14 pp. MR3056077

[R17] Radon, J., Uber die Bestimmung von Funktionen durch ihre Integralwerte längs gewisser Mannigfaltigkeiten, Ber. Saechs Akad. Wiss. Leipzig, Math.-Phys. 69 (1917), 262-267.

[Sh92] Sharafutdinov, V. A., On the problem of emission tomography for nonhomogeneous media, Dokl. Akad. Nauk 326 (1992), 446-448. (Russian). English transl.: Soviet Phys. Dokl. 37 (1992) 469-470. MR1203226

[Sh93] Sharafutdinov, V. A., Uniqueness theorems for the exponential X-ray trans- 
form, J. Inverse Ill-Posed Probl. 1 (1993), 355-372. MR1256128

[TM80] Tretiak, O. J. and Metz, C., The exponential Radon transform, SIAM J. Appl. Math. 39 (1980), 341-354. MR0588505

[Q83] Quinto, E. T., The invertibility of rotation invariant Radon transforms, $J$. Math. Anal. Appl. 91 (1983), 510-522. MR0690884

F. O. Goncharov

CMAP, CNRS, Ecole Polytechnique

Institut Polytechnique de Paris

91128 Palaiseau

France

fedor.goncharov.ol@gmail.com
R. G. Novikov

CMAP, CNRS, Ecole Polytechnique

Institut Polytechnique de Paris

91128 Palaiseau

France

and

IEPT RAS

117997 Moscow

Russia

roman.novikov@polytechnique.edu

Received March 22, 2019 